\title{
Design and simulation analysis of non-receiver aware and receiver aware multicast location prediction based routing protocols for mobile ad hoc networks
}

\author{
Natarajan Meghanathan \\ Department of Computer Science, Jackson State University, Jackson, MS, USA \\ E-mail: natarajan.meghanathan@jsums.edu, Tel +1-601-979-3661, Fax.+1-601-979-2478
}

\begin{abstract}
We present multicast extensions to our recently proposed Location Prediction Based Routing (LPBR) protocol, referred to as NR-MLPBR and R-MLPBR, to simultaneously reduce the number of multicast tree discoveries and number of links per tree and/or the hop count per source-receiver path in mobile ad hoc networks (MANETs). Nodes running NR-MLPBR are not aware of the receivers of the multicast group; R-MLPBR assumes each receiver node knows the identity of the other receivers of the group. Upon failure of a path to the source, a receiver attempts to locally construct a global topology using the location and mobility information collected during the latest broadcast tree discovery. NR-MLPBR predicts a path that has the minimum number of hops to the source and R-MLPBR predicts a path to the source that has the minimum number of non-receiver nodes. If the predicted path exists in the network at that time instant, the source accommodates the path as part of the multicast tree and continues to send multicast packets in the modified tree without attempting a broadcast discovery. Simulation results show that NR-MLPBR and R-MLPBR perform better than the minimum-hop based and minimum-link based on-demand multicast MANET routing protocols under different conditions of node mobility, density and group size.
\end{abstract}

Keywords: Multicast Routing, Mobile Ad hoc Networks, Location Prediction, Link Efficiency, Hop Count, Simulation

\section{Introduction}

A mobile ad hoc network (MANET) is a dynamic distributed system of wireless nodes that move independent of each other in an autonomous fashion. The network bandwidth is limited and the medium is shared. As a result, transmissions are prone to interference and collisions. The battery power of the nodes is constrained and hence nodes operate with a limited transmission range, often leading to multi-hop routes between any pair of nodes in the network. Due to node mobility, routes between any pair of nodes frequently change and need to be reconfigured. As a result, on-demand route discovery (discovering a route only when required) is often preferred over periodic route discovery and maintenance, as the latter strategy will incur significant overhead due to the frequent exchange of control information among the nodes (Broch et al., 1998). We hence deal with on-demand routing protocols for the rest of this paper.

Multicasting is the process of sending a stream of data from one source node to multiple recipients by establishing a routing tree, which is an acyclic connected subgraph containing all the nodes in the tree. The set of receiver nodes form the multicast group. While propagating down the tree, data is duplicated only when necessary. This is better than multiple unicast transmissions. Multicasting in ad hoc wireless networks has numerous applications (Toh et al., 2000): collaborative and distributing computing like civilian operations, emergency search and rescue, law enforcement, warfare situations and etc.

Several MANET multicast routing protocols have been proposed in the literature (Murthy and Manoj, 2004). They are mainly classified as: tree-based and mesh-based protocols. In tree-based protocols, only one route exists between a source and a destination and hence these protocols are efficient in terms of the number of link transmissions. The tree-based protocols can be further divided into two types: source tree-based and shared tree-based. In source tree-based multicast protocols, the tree is rooted at the source. In shared tree-based multicast protocols, the tree is rooted at a core node and all communication between the 
multicast source and the receiver nodes is through the core node. Even though shared tree-based multicast protocols are more scalable with respect to the number of sources, these protocols suffer under a single point of failure, the core node. On the other hand, source tree-based protocols are more efficient in terms of traffic distribution. In mesh-based multicast protocols, multiple routes exist between a source and each of the receivers of the multicast group. A receiver node receives several copies of the data packets, one copy through each of the multiple paths. Mesh-based protocols provide robustness at the expense of a larger number of link transmissions leading to inefficient bandwidth usage. A detailed classification tree of the different classes of multicast routing protocols is illustrated in Figure 1. Considering all the pros and cons of these different classes of multicast routing in MANETs, we feel the source tree-based multicast routing protocols are more efficient in terms of traffic distribution and link usage. Hence, all of our work in this research will be in the category of on-demand source tree-based multicast routing.

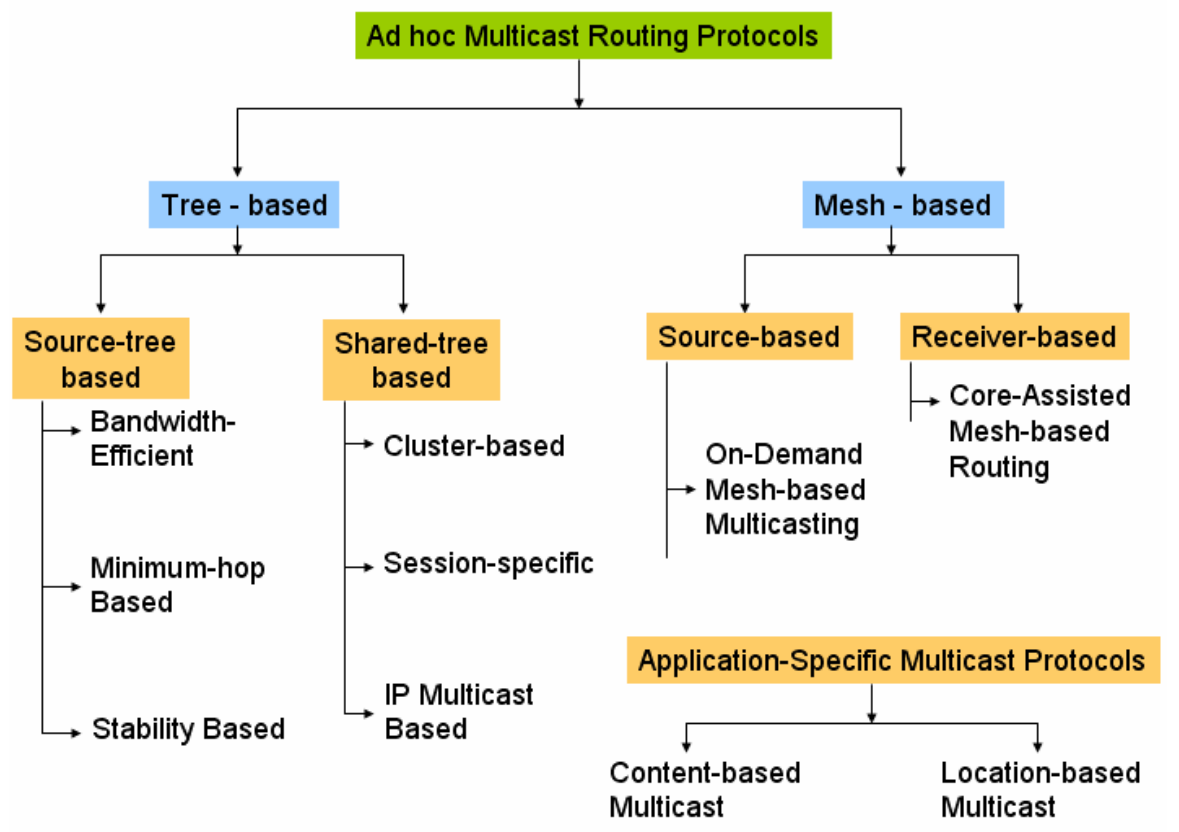

Figure 1. Classification of Ad hoc Multicast Routing Protocols

Within the class of on-demand source tree-based routing protocols, three categories of multicast routing protocols have been identified (i) Bandwidth-efficient protocols that aim to minimize the total number of links in the tree; (ii) Minimum-hop based protocols that aim to minimize the number of hops in the paths from the source to every receiver node and (iii) Stability-based protocols that aim to determine long-living stable trees and reduce the time between successive global tree discoveries. The Bandwidth-Efficient Multicast Routing Protocol (BEMRP, Ozaki et al., 2001), Multicast Extension to the Ad hoc On-demand Distance Vector (MAODV) routing protocol (Royer and Perkins, 1999) and the Associativity-Based Ad hoc Multicast (ABAM, Toh et al., 2000) routing protocols are classical examples of the bandwidth-efficient, minimum-hop based and the stability-based multicast protocol categories. Meghanathan (2008) conducted a detailed performance study of these three multicast routing protocols. Simulation study results reveal that MAODV trees are highly unstable, but have an average hop count close to the minimum number of hops between the source and the receivers (Meghanathan, 2008). BEMRP discovers trees that have a reduced number of links but have a higher average hop count per source-receiver path. ABAM discovers trees that are stable, but have a higher average hop count per source-receiver path as well as higher number of links per tree compared to BEMRP. A significant observation by Meghanathan (2008) is that BEMRP trees are as stable as the trees discovered using ABAM. This can be attributed to the reduced number of links in the trees determined by BEMRP, leading to longer lifetime of the trees. Because of these observations, we use only MAODV and BEMRP in our simulation studies conducted in this paper.

Meghanathan (2009a) developed a location prediction based routing (LPBR) protocol for unicast routing in MANETs. The specialty of LPBR is that it attempts to simultaneously reduce the number of global broadcast route discoveries as well as the hop count of the paths for a source-destination session. LPBR works as follows: During a regular flooding-based route discovery, LPBR collects the location and mobility information of the nodes in the network and stores the collected information at the destination node of the route search process. When the minimum-hop route discovered through the flooding-based route discovery fails, the destination node attempts to predict the current location of each node using the location and mobility information collected during the latest flooding-based route discovery. A minimum hop path Dijkstra algorithm (Cormen et al., 2001) is run on the locally predicted global topology. If the predicted minimum hop route exists in the network at that time instant, no expensive flooding-based route discovery is needed and the source continues to send data packets on the discovered route; otherwise, the source initiates another flooding-based route discovery. 
In this paper, we propose two multicast extensions to LPBR, referred to as NR-MLPBR and R-MLPBR. Both the multicast extensions are aimed at minimizing the number of global broadcast tree discoveries as well as the hop count per source-receiver path of the multicast tree. They use a similar idea of letting the receiver nodes to predict a new path based on the locally constructed global topology obtained from the location and mobility information of the nodes learnt through the latest broadcast tree discovery. Receiver nodes running NR-MLPBR (Non-Receiver aware Multicast extensions of LPBR) are not aware of the receivers of the multicast group, whereas each receiver node running R-MLPBR (Receiver-aware Multicast Extension of LPBR) is aware of the identity of the other receivers of the multicast group. NR-MLPBR attempts to predict a minimum hop path to the source, whereas R-MLPBR attempts to predict a path to the source that has the minimum number of non-receiver nodes. If more than one path has the same minimum number of non-receiver nodes, then R-MLPBR breaks the tie among such paths by choosing the path with the minimum number of hops to the source. Thus, R-MLPBR is also designed to reduce the number of links in the multicast tree, in addition to the average hop count per source-receiver path and the number of global broadcast tree discoveries.

The rest of the paper is organized as follows: In Section 2, we provide a brief overview of the two multicast extensions to LPBR. Section 3 provides the detailed design of the two multicast extensions. Section 4 explains the simulation environment and reviews the MAODV and BEMRP protocols that are studied along with NR-MLPBR and R-MLPBR as part of our simulation studies. Global tree discoveries are performed using the default commonly used approach of flooding and our recently proposed density and mobility aware energy-efficient flooding strategy called DMEF (Meghanathan, 2009b). In Section 5, we illustrate and explain simulation results for the four multicast routing protocols (MAODV, NR-MLPBR, R-MLPBR and BEMRP) with respect to different performance metrics under both flooding and DMEF. Section 6 concludes the paper.

\section{Basic Idea of the Multicast Extensions to LPBR}

The multicast extensions of LPBR work as follows: When a source attempts to construct a multicast tree, it floods a Multicast Tree Request Message (MTRM) throughout the network. The location and mobility information of the intermediate forwarding nodes are recorded in the MTRM. Each node, including the receiver nodes of the multicast group, broadcasts the MTRM exactly once in its neighborhood. Each receiver node of the multicast group receives several MTRMs and sends a Multicast Tree Establishment Message (MTEM) on the minimum hop path traversed by the MTRMs. The set of paths traversed by the MTEMs form the multicast tree rooted at the source.

If an intermediate node of the tree notices a downstream node moving away from it, the intermediate node sends a Multicast Path Error Message (MPEM) to the source node. The source node does not immediately initiate another tree discovery procedure. Instead, the source node waits for the appropriate receiver node (whose path to the source has broken) to predict a path to the source. The receiver node predicts a new path based on the location and mobility information of the nodes collected through the MTRMs during the latest global tree discovery procedure. The receiver node attempts to locally construct the global topology by predicting the locations of the nodes in the network using the latest location and mobility information collected about the nodes.

NR-MLPBR and R-MLPBR differ from each other based on the type of path predicted and notified to the source. NR-MLPBR determines the minimum hop path to the source and sends a Multicast Predicted Path Message (MPPM) on the minimum hop path to the source. R-MLPBR assumes that each receiver knows the identity of the other receivers of the multicast group (learnt through the latest broadcast tree discovery process) and hence attempts to choose a path that will minimize the number of newly added intermediate nodes to the multicast tree. In pursuit of this, R-MLPBR determines a set of node-disjoint paths to the source on the predicted topology and sends the MPPM on that path that includes the minimum number of non-receiver nodes. If there is a tie, RMLPBR chooses the path that has the least hop count. The source waits to receive a MPPM from the affected receiver node. If a MPPM is received within a certain time, the source considers the path traversed by the MPPM as part of the multicast tree and continues to send the data packets down the tree including to the nodes on the new path. Otherwise, the source initiates another global tree discovery procedure by broadcasting the MTRM. R-MLPBR has been thus designed to also reduce the number of links that form the multicast tree, in addition to the source-receiver hop count and the number of global tree discoveries. Nevertheless, as observed in our simulations, R-MLPBR cannot completely nullify the tradeoff between the hop count per source-receiver path and the number of links in the tree.

\section{Detailed Design of the Multicast Extensions to LPBR}

The objective of the multicast extensions to LPBR (referred to as NR-MLPBR and R-MLPBR) is to simultaneously minimize the number of global broadcast tree discoveries as well as the hop count per source-receiver path. The Non-Receiver aware Multicast extension to LPBR (NR-MLPBR) precisely does this and it does not assume the knowledge of the receiver nodes of the multicast group at every receiver node. The Receiver-aware multicast extension of LPBR (R-MLPBR) assumes that each receiver node knows the identities of the other receiver nodes in the multicast group. This enables R-MLPBR to also reduce the number of links in the multicast tree in addition to reducing the number of global broadcast tree discoveries and the hop count per sourcereceiver path. Each receiver node running R-MLPBR learns the identity information of peer receiver nodes through the broadcast tree discovery procedure. Both the multicast extensions assume the periodic exchange of beacons in the neighborhood. This is essential for nodes to learn about the moving away of the downstream nodes in the multicast tree. The following sections describe 
the working of the two multicast extensions in detail. Unless otherwise stated specifically, the description holds good for the both NR-MLPBR and R-LPBR. We also assume that a multicast group comprises basically of receiver nodes that wish to receive data packets from an arbitrary source, which is not part of the multicast group.

3.1 Broadcast of Multicast Tree Request Messages: Whenever a source node has data packets to send to a multicast group and is not aware of a multicast tree to the group, the source initiates a broadcast tree discovery procedure by broadcasting a Multicast Tree Request Message (MTRM) to its neighbors. The source maintains a monotonically increasing sequence number for the broadcast tree discoveries it initiates to form the multicast tree. Each node, including the receiver nodes of the multicast group, on receiving the first MTRM of the current broadcast process (i.e., a MTRM with a sequence number greater than those seen before), includes its Location Update Vector, LUV in the MTRM packet. The LUV of a node comprises the following: node ID, X, Y coordinate information, Is Receiver flag, Current velocity and Angle of movement with respect to the X-axis. The Is Receiver flag in the LUV, if set, indicates that the node is a receiving node of the multicast group. The node ID is also appended on the "Route record" field of the MTRM packet. The structure of the LUV and the MTRM is shown in Figures 2 and 3 respectively.

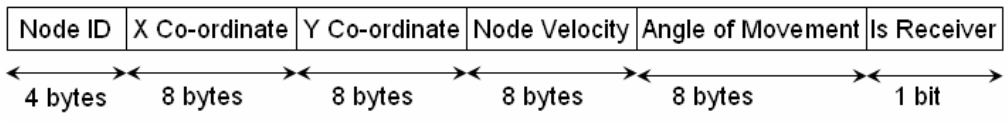

Figure 2. Location Update Vector (LUV) Collected from Each Node

\begin{tabular}{|c|c|c|c|c|}
\hline $\begin{array}{c}\text { Multicast } \\
\text { Source }\end{array}$ & $\begin{array}{l}\text { Multicast } \\
\text { Group ID }\end{array}$ & $\begin{array}{c}\text { Sequence } \\
\text { Number }\end{array}$ & $\begin{array}{l}\text { Route Recorded } \\
\text { (List of Node IDs) }\end{array}$ & $\begin{array}{l}\text { Location Update } \\
\text { Vectors (LUVs) }\end{array}$ \\
\hline 4 bytes & 4 bytes & 4 bytes & $\begin{array}{c}\text { Variable Size } \\
\text { of } 4 \text { bytes }\end{array}$ & $\begin{array}{c}\text { Variable Size } \\
\text { of } 36 \text { bytes, } 1 \text { bit }\end{array}$ \\
\hline
\end{tabular}

Figure 3. Structure of the Multicast Tree Request Message (MTRM)

3.2 Construction of the Multicast Tree through the Multicast Tree Establishment Message: Paths constituting the multicast tree are independently chosen at each receiver node. A receiver node gathers several MTRMs obtained across different paths and selects the minimum hop path among them by looking at the "Route Record" field in these MTRMs. A Multicast Tree Establishment Message (MTEM) is sent on the discovered minimum hop route to the source. The MTEM originating from a receiver node has the list of node IDs corresponding to the nodes that are on the minimum hop path from the receiver node to the source (which is basically the reverse of the route recorded in the MTRM). The structure of the MTEM packet is shown in Figure 4.

\begin{tabular}{|c|c|c|c|c|}
\hline $\begin{array}{c}\text { Multicast } \\
\text { Source }\end{array}$ & $\begin{array}{l}\text { Originating } \\
\text { Receiver }\end{array}$ & $\begin{array}{l}\text { Multicast } \\
\text { Group ID }\end{array}$ & $\begin{array}{l}\text { Sequence } \\
\text { Number }\end{array}$ & $\begin{array}{l}\text { Route Record from the } \\
\text { Receiver to the Source }\end{array}$ \\
\hline 4 bytes & 4 bytes & 4 bytes & 4 bytes & $\begin{array}{l}\text { Variable Size } \\
\text { of } 4 \text { bytes }\end{array}$ \\
\hline
\end{tabular}

Figure 4. Structure of the Multicast Tree Establishment Message (MTEM)

\begin{tabular}{|c|c|c|c|c|}
\hline Key & \multicolumn{3}{|c|}{ Value } \\
\hline$<$ Source, Multicast Group ID $>$ & $\left\langle\mathrm{d}_{\mathrm{a}}, \mathrm{r}_{\mathrm{a}}\right\rangle$ & $\left\langle\mathrm{d}_{\mathrm{b}}, \mathrm{r}_{\mathrm{b}}\right\rangle$ & $\langle,,, \ldots\rangle$ & $<\ldots, \ldots\rangle$ \\
\hline
\end{tabular}

Figure 5. Structure of the Multicast Routing Table at an Intermediate Node

An intermediate node upon receiving the MTEM packet checks its multicast routing table whether there exist an entry for the $<$ Multicast Source, Multicast Group ID $>$ in the table. The multicast routing table at a node is an ordered entry of $\langle k e y\rangle\langle v a l u e\rangle$ pairs, where the key is the tuple $<$ Multicast Source, Multicast Group ID $>$ and the value is the tuple $<$ Downstream node, Receiver node $>$. The set of downstream nodes are part of the multicast tree rooted at the source node for the multicast group. If an entry exists, the intermediate node merely adds the tuple < One-hop sender of the MTEM, Originating Receiver node of the MTEM $>$ to the list of $<$ Downstream node, Receiver node $>$ tuples for the multicast tree entry and does not forward the MTEM further. If a $<$ Multicast Source, Multicast Group ID $>$ entry does not exist in the multicast routing table, the intermediate node creates an entry and initializes it with the <One-hop sender of the MTEM, Originating Receiver node of the MTEM $>$ tuple. Note that the one-hop sender of the MTEM is learnt through the MAC (Medium Access Control) layer header and verified using the Route Record field in the MTEM. The intermediate node then forwards the MTEM to the next downstream node on the path towards the source. The structure of the multicast routing table at a node is illustrated in Figure 5. Note that the tuples $\left\langle d_{a}, r_{a}\right\rangle,\left\langle d_{b}, r_{b}\right\rangle,\langle\ldots, \ldots\rangle$ indicate the downstream node $d_{a}$ for receiver node $r_{a}$, downstream node $d_{b}$ for receiver node $r_{b}$ and so on. A node could be the downstream 
node for more than one receiver node. Figure 6 shows an example of the multicast routing table established at some of the intermediate nodes for a multicast tree rooted at source node with ID 0 and multicast group with ID M1.

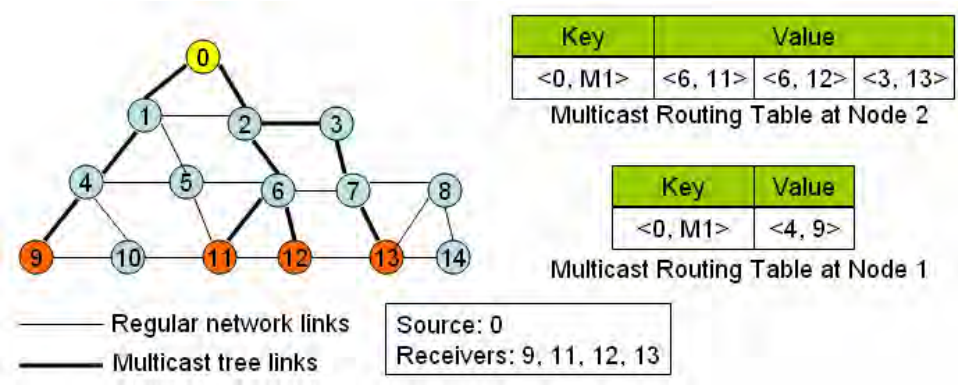

Figure 6. Example for Multicast Routing Table Established at Intermediate Nodes

The source node maintains a multicast routing table that has the list of $<$ Downstream node, Receiver node $>$ tuples for each of the multicast groups to which the source is currently communicating through a multicast session. For each MTEM received, the source adds the neighbor node that sent the MTEM and the corresponding Originating Receiver node to the list of $<$ Downstream node, Receiver node> tuples for the multicast group.

3.3 Multicast Tree Acquisition Time and Data Transmission: After receiving the MTEMs from all the receiver nodes within a certain time called the Tree Acquisition Time (TAT), the source starts sending the data packets on the multicast tree. The TAT is based on the maximum possible diameter of the network (an input parameter in our simulations). The diameter of the network is the maximum of the hop count of the minimum hop paths between any two nodes in the network. The TAT is dynamically set at a node depending on the time it took to receive the first MTEM for a broadcast tree discovery procedure. If perMulticastPeriod denotes the time between the transmission of successive multicast packets from the source, delFirstMTEMRecvd indicates the time lapsed between the initiation of the MTRM broadcast and the receipt of the first MTEM and hopsFirstMTEMRecvd denotes the number of hops traversed by the first MTEM received, then,

$$
\text { TAT }=\text { Minimum }\left[\text { perMulticastPeriod },\left(\frac{\text { delFirstMTEM Re } c v d * \text { Diameter }}{\text { hopsFirstMTEM Recvd }}\right)\right]
$$

We assume the source at least knows the multicast group size, if not the identification information for each of the receivers of the multicast group. Hence, if the source fails to receive the required number of MTEMs (equal to the multicast group size), within the TAT, the source initiates another global broadcast tree discovery procedure. If the source receives the MTEMs from all the receivers, equaling to the multicast group size, the source starts sending the data packets down the multicast tree.

The structure of the header of the multicast data packet is shown in Figure 7. The source and destination fields in the header include the identification for the source node and the multicast group ID respectively. The sequence number field in the header can be used by the receivers to accumulate and reorder the multicast data packets, incase if they are received out of order. In addition to these regular fields, the header of the multicast data packet includes three specialized fields: the 'More Packets' (MP) field, the 'Current Dispatch Time' (CDT) field and the 'Time Left for Next Dispatch' (TLND) field. The CDT field stores the time as the number of milliseconds lapsed since Jan 1, 1970, 12 AM. These additional overhead (relative to that of the other ad hoc multicast routing protocols) associated with the header of each data packet amounts to only 12 more bytes per data packet.

\begin{tabular}{|l|c|c|c|c|c|}
$\begin{array}{c}\text { Multicast } \\
\text { Source }\end{array}$ & $\begin{array}{c}\text { Multicast } \\
\text { Group ID }\end{array}$ & $\begin{array}{c}\text { Sequence } \\
\text { Number }\end{array}$ & $\begin{array}{c}\text { More } \\
\text { Packets }\end{array}$ & $\begin{array}{c}\text { Current } \\
\text { Dispatch Time }\end{array}$ & $\begin{array}{c}\text { Time Left for } \\
\text { Next Dispatch }\end{array}$ \\
$\stackrel{4}{4 \text { bytes }}$ \\
Figure 7. Structure of the Header of the Multicast Data Packet
\end{tabular}

The source sets the $C D T$ field in all the data packets sent. In addition, if the source has any more data to send, it sets the MP flag to 1 and sets the appropriate value for the TLND field (equal to perMulticastPeriod), which indicates the number of milliseconds since the CDT. If the source does not have any more data to send, it will set the MP flag to 0 and leaves the TLND field blank. As we assume the clocks across all nodes are synchronized, a receiver node will be able to calculate the end-to-end delay for the data packet based on the time the data packet reaches the node and the CDT field in the header of the data packet. Several clock synchronization algorithms (example: Naumov et al., 2006; Sheu et al., 2007) have been proposed for wireless ad hoc networks. The receiver node computes and maintains the average end-to-and delay per data packet for the current path to the source by recording the sum of the end-to-end delays of all the data packets received so far on the path and the number of data packets 
received on the path. Accordingly, the average end-to-end delay per data packet for the current path is updated every time after receiving a new data packet on the path. If the source node has set the MP flag, the receiver node computes the 'Next Expected Packet Arrival Time' (NEPAT), which is CDT field + TLND field + 2*Average end-to-end delay per data packet. A timer is started for the NEPAT value. Since, we are using only the average end-to-end delay per data packet to measure the NEPAT value, the variations in the end-to-end delay of particular data packets will not very much affect the NEPAT value. So, the source and receiver nodes need not be perfectly synchronized. The clocks across the nodes can have small drifts and this would not very much affect the performance of the multicast extensions of LPBR.

3.4 Multicast Tree Maintenance: We assume that each node periodically exchanges beacon messages with its neighbors, located within its default maximum transmission range. If an intermediate node notices that its link with a downstream node has failed (i.e., the two nodes have moved away and are no longer neighbors), the intermediate node generates and sends a Multicast Path Error Message (MPEM) to the source node of the multicast group entry. The MPEM has information about the receiver nodes affected (obtained from the multicast routing table) because of the link failure with the downstream node. The structure of the MPEM is shown in Figure 8. The intermediate node removes the tuple(s) corresponding to downstream node(s) and the affected receiver node(s). After these deletions, if no more $<$ Downstream node, Receiver node $>$ tuple exists for a $<$ Source node, Multicast group ID> key entry, the intermediate node removes the entire row for this entry from the multicast routing table.

\begin{tabular}{|c|c|c|c|}
\hline $\begin{array}{c}\text { Multicast } \\
\text { Source }\end{array}$ & \begin{tabular}{|c|} 
Originating \\
Intermediate Node
\end{tabular} & $\begin{array}{l}\text { Multicast } \\
\text { Group ID }\end{array}$ & $\begin{array}{c}\text { IDs of } \\
\text { Affected Receivers }\end{array}$ \\
\hline & & & $\begin{array}{c}\text { Variable Size } \\
\text { of } 4 \text { bytes }\end{array}$ \\
\hline
\end{tabular}

Figure 8. Structure of a MPEM Message

The source node, upon receiving the MPEM, will wait to receive a Multicast Predicted Path Message (MPPM) from each of the affected receivers, within a MPPM-timer maintained for each receiver. The source node estimates a Tree-Repair Time (TRT) for each receiver as the time that lapsed between the reception of the MPEM from an intermediate node and the MPPM from the affected receiver. An average value for the TRT per receiver is maintained at the source as it undergoes several path failures and repairs before the next global broadcast based tree discovery. The MPPM-timer (initially set to the time it took for the source to receive the MTEM from the receiver) for a receiver will be then set to 1.5* Average TRT value, so that we give sufficient time for the destination to learn about the route failure and generate a new MPPM. Nevertheless, this timer will be still far less than the tree acquisition time that would be incurred if the source were to launch a global broadcast tree discovery. Hence, our approach will only increase the network throughput and does not decrease it.

3.5 Prediction of Node Location using the Location Update Vector: If a receiver node does not receive the data packet within the NEPAT time, it will attempt to locally construct the global topology using the location and mobility information of the nodes learnt from the latest broadcast tree discovery. Each node is assumed to be continuing to move in the same direction with the same speed as mentioned in its latest LUV. Based on this assumption and information from the latest LUVs, the location of each node at the NEPAT time is predicted. Whenever a node changes its direction, we assume the node is moving in the new direction with a particular velocity and towards a particular targeted destination location. As a result, a node can determine its angle of movement with respect to the X-axis at time STIME by computing the slope of the line joining the current location co-ordinates of the node at time STIME and the co-ordinates of the targeted location to which the node is moving. After reaching the targeted location, a node can change its velocity and direction to move to a new destination location.

We now explain how to predict the location of a node (say node $u$ ) at a time instant CTIME based on the LUV gathered from node $u$ at time STIME. Let $\left(X_{u}^{\text {STIME }}, Y_{u}^{\text {STIME }}\right)$ be the $\mathrm{X}$ and $\mathrm{Y}$ co-ordinates of node $u$ at time STIME. Let Angle ${ }_{u}^{\text {STIME }}$ and Velocity $_{u}{ }^{\text {STIME }}$ represent the angle of movement with respect to the $\mathrm{X}$-axis and the velocity at which node $u$ is moving. The distance traveled by node $u$ from time STIME to CTIME would be: Distance $_{u}{ }^{\text {STIME-CTIME }}=(\text { CTIME }- \text { STIME }+1)^{*}$ Velocity ${ }_{u}^{\text {STIME }}$.

Let $\left(X_{u}^{\text {CIIME }}, Y_{u}{ }^{\text {CTIME }}\right)$ be the predicted location of node $u$ at time CTIME. The value of $X_{u}^{{ }^{C T I M E}}$ is given by $X_{u}^{\text {STIME }}+$ Offset$X_{u}{ }^{C T I M E}$ and the value of $Y_{u}^{C T I M E}$ is given by $Y_{u}^{\text {STIME }}+$ Offset- $Y_{u}^{\text {CTIME }}$. The offsets in the $\mathrm{X}$ and Y-axes, depend on the angle of movement and the distance traveled, and are calculated as follows:

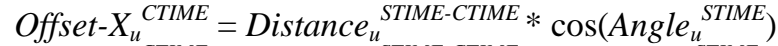

$$
\begin{aligned}
& \text { Offset- } Y_{u}^{\text {CTIME }}=\text { Distance }_{u}{ }_{\text {STIME-CTIME }} * \sin \left(\text { Angle }_{u}{ }^{\text {STIME }}\right) \\
& X_{u}^{\text {CTIME }}=X_{u}^{\text {STIME }}+\text { Offset- } X_{u}^{\text {CTIME }} \\
& Y_{u}^{\text {CTIME }}=Y_{u}^{\text {STIME }}+\text { Offset }-Y_{u}{ }^{\text {CTIME }}
\end{aligned}
$$

We assume each node is initially configured with information regarding the network boundaries, given by [0, 0], $\left[X_{\max }, 0\right],\left[X_{\max }\right.$, $\left.Y_{\max }\right]$ and $\left[0, Y_{\max }\right]$. When the predicted $\mathrm{X}$ and/or $\mathrm{Y}$ co-ordinate is beyond the network boundaries, we set their values to the boundary conditions as stated below. 


$$
\begin{aligned}
& \text { If }\left(X_{u}^{\text {CTIME }}<0\right) \text {, then } X_{u}^{\text {CTIME }}=0 \text {; If }\left(X_{u}^{\text {CTIME }}>X_{\max }\right) \text {, then } X_{u}^{\text {CTIME }}=X_{\max } \\
& \text { If }\left(Y_{u}^{\text {CTIME }}<0\right) \text {, then } Y_{u}^{\text {CTIME }}=0 \text {; If }\left(Y_{u}^{\text {CTIME }}>Y_{\max }\right) \text {, then } Y_{u}^{\text {CTIME }}=Y_{\max }
\end{aligned}
$$

Based on the predicted locations of each node in the network at time CTIME, the receiver node locally constructs the global topology. Note that there exists an edge between two nodes in the locally constructed global topology, if the predicted distance between the two nodes (with the location information obtained from the LUV) is less than or equal to the transmission range of the nodes. The two multicast extensions NR-MLPBR and R-MLPBR differ from each other on the nature of the paths predicted at the receiver node. In Section 3.6, we discuss the predicted path selection procedure in the case of NR-MLPBR. Section 3.7 discusses the algorithm used in the selection of the predicted paths in the case of R-MLPBR.

3.6 NR-MLPBR: Multicast Path Prediction: The receiver node locally runs the Dijkstra's minimum hop path algorithm (Cormen et al., 2001) on the predicted global topology. If at least one path exists from the source node to the receiver node in the generated topology, the algorithm returns the minimum hop path among them. The receiver node then sends a MPPM (structure shown in

\begin{tabular}{|c|c|c|c|}
\hline $\begin{array}{l}\text { Multicast } \\
\text { Source }\end{array}$ & $\begin{array}{l}\text { Originating } \\
\text { Receiver Node }\end{array}$ & $\begin{array}{l}\text { Multicast } \\
\text { Group ID }\end{array}$ & $\begin{array}{l}\text { Predicted Path to the Multicast } \\
\text { Source (List of Node IDs) }\end{array}$ \\
\hline 4 bytes & 4 bytes & 4 bytes & Variable Size of \\
\hline
\end{tabular}
Figure 9) on the discovered path with the route information included in the message.

Figure 9. Structure of the Multicast Predicted Path Message (MPPM)

3.7 R-MLPBR: Multicast Path Prediction: The receiver node uses the LUV obtained from each of the intermediate nodes during the latest global tree broadcast discovery process to learn about the identification (IDs) of its peer receiver nodes that are part of the multicast group. If there existed a direct path to the source on the predicted topology, the receiver node chooses that path as the predicted path towards the source. Otherwise, the receiver node determines a set of node-disjoint paths on the predicted global topology. The node-disjoint paths to the source are ranked depending on the number of non-receiver nodes that act as intermediate nodes on the path. The path that has the least number of non-receiver nodes as intermediate nodes is preferred. The reason is a path that has the least number of non-receiver nodes is more likely to be a minimum hop path and if a receiver node lies on that path, the number of newly added links to the tree would also be reduced. R-MLPBR thus aims to discover paths with the minimum hop count and at the same time attempts to conserve bandwidth by reducing the number of links that get newly added to the tree as a result of using the predicted path. The MPPM is hence sent on the predicted path that has minimum number of non-receiver nodes. If two or more paths has the same minimum number of non-receiver nodes, R-MLPBR breaks the tie by choosing the path with the minimum hop count to the source. Figure 10 illustrates the algorithm used by R-MLPBR at a receiver node to select the best predicted path to the source.

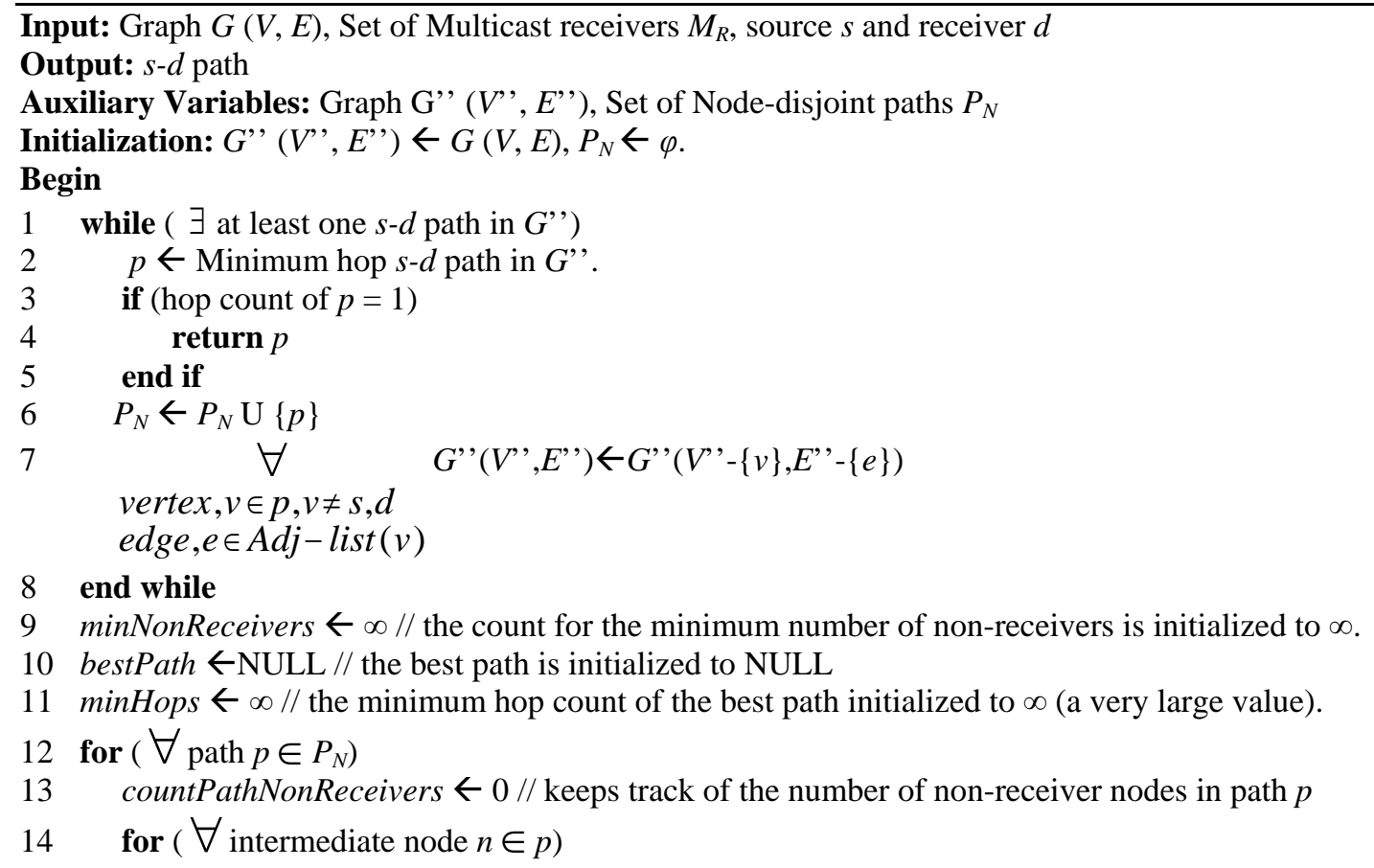




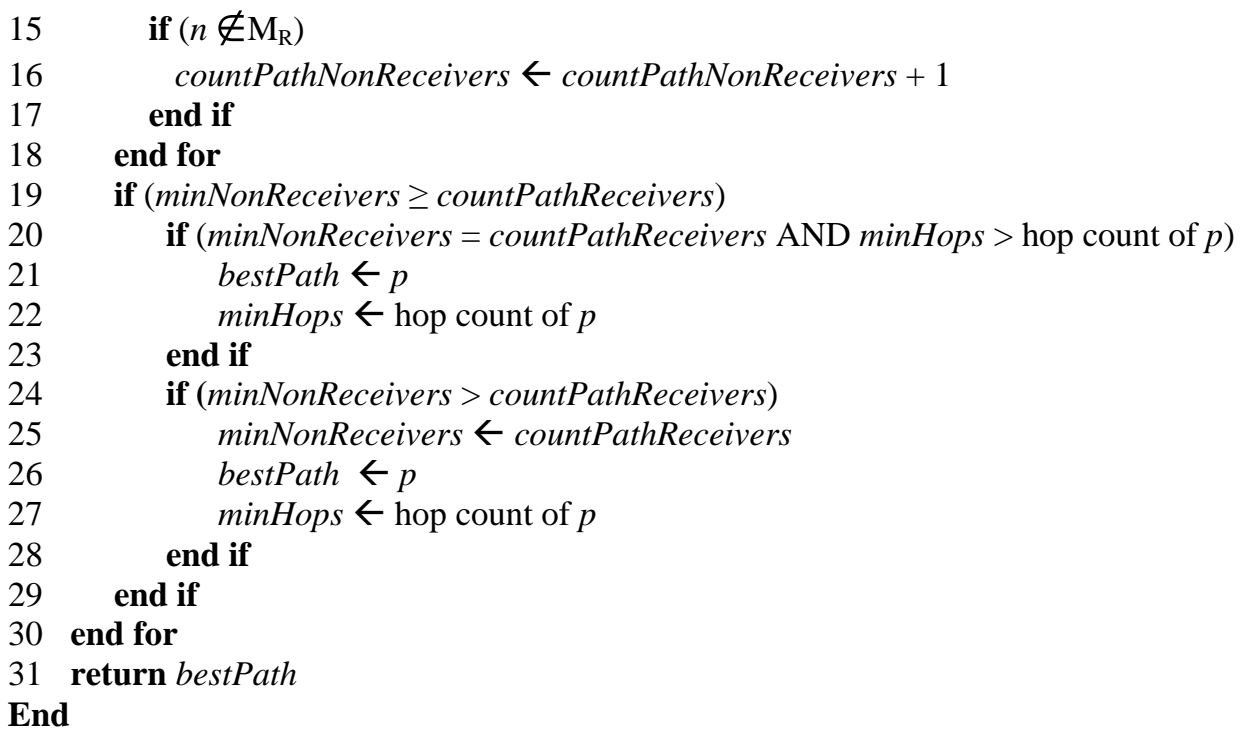

Figure 10. R-MLPBR Predicted Path Selection Algorithm

Note that we designed R-MLPBR to choose the path with the minimum number of non-receiver nodes, rather than the path with the maximum number of receiver nodes, as the latter design has the possibility of yielding paths with significantly larger hop count from the source to the receiver node without any guarantee on the possible reduction in the number of links. Our design of choosing the path with the minimum number of non-receiver nodes helps to maintain the hop count per source-receiver path close to that of the minimum hop count and at the same time does helps to reduce the number of links in the tree to a certain extent.

3.8 Propagation of the Multicast Predicted Path Message towards the Source: An intermediate node on receiving the MPPM, checks its multicast routing table if there already exists a key entry for the source node and the multicast group to which the MPPM belongs to. If an entry exists, the intermediate node merely adds the tuple <One-hop sender of the MPPM, Originating Receiver node of the MPPM $>$ to the list of <Downstream node, Receiver node $>$ tuples for the multicast tree entry. If the $<$ Multicast Source, Multicast Group ID $>$ entry does not exist in the multicast routing table, the intermediate node creates an entry and initializes it with the <One-hop sender of the MPPM, Originating Receiver node of the MPPM> tuple. In either case, the MPPM is then forwarded to the next downstream node on the path towards the source. If the source node receives the MPPM from the appropriate receiver node before the MPPM-timer expires, it indicates that the predicted path does exist in the network at that time instant. A costly global broadcast tree discovery has been thus avoided. The source continues to send the data packets down the multicast tree. The source node estimates the Tree Repair Time (TRT) as the time lapsed between the reception of the MPEM from an intermediate node and the MPPM from the appropriate receiver node. An average TRT value for each receiver is thus maintained at the source as it undergoes several route failures and repairs before the next global broadcast-based tree discovery.

3.9 Handling Prediction Failure: If an intermediate node attempting to forward the MPPM of a receiver node could not successfully forward the packet to the next node on the path towards the source, the intermediate node informs the absence of the route through a MPPM-Error packet (structure shown in Figure 11) sent back to the receiver node. The receiver node on receiving the MPPM-Error packet discards all the LUVs and does not generate any new MPPM. The receiver will wait for the multicast source to initiate a global broadcast-based tree discovery. After the MPPM-timer expires, the multicast source initiates a new global broadcast-based tree discovery procedure.

\begin{tabular}{|c|c|c|c|c|}
\hline $\begin{array}{l}\text { Node Sending the } \\
\text { MPPM-Error Packet }\end{array}$ & $\begin{array}{c}\text { Multicast } \\
\text { Source }\end{array}$ & $\begin{array}{c}\text { Originating } \\
\text { Receiver Node }\end{array}$ & $\begin{array}{l}\text { Multicast } \\
\text { Group ID }\end{array}$ & $\begin{array}{l}\text { Sequence No. } \\
\text { of latest MTRM }\end{array}$ \\
\hline 4 bytes & 4 bytes & 4 bytes & 4 bytes & 4 bytes \\
\hline
\end{tabular}

Figure 11. Structure of the MPPM-Error Packet

\section{Simulation Environment, Review of MANET Multicast Routing Protocols and Performance Metrics}

The network dimension used is a $1000 \mathrm{~m}$ x $1000 \mathrm{~m}$ square network. The transmission range of each node is assumed to be $250 \mathrm{~m}$. The number of nodes used in the network is 25, 50 and 75 nodes representing networks of low, medium and high density with an average distribution of 5, 10 and 15 neighbors per node respectively. Initially, nodes are uniformly randomly distributed in the 
network. We compare the performance of NR-MLPBR and R-MLPBR with that of the minimum-hop based MAODV and the link-efficient BEMRP protocols. We implemented all of these four multicast routing protocols in ns-2 (Breslau et al., 2000). The broadcast tree discovery strategies simulated are the default flooding approach and our recently proposed density and mobility aware energy-efficient broadcast strategy called DMEF. The simulation conditions are summarized in Table 1.

Table 1. Simulation Environment (Conditions and Models)

\begin{tabular}{|c|c|c|}
\hline Network Size & \multicolumn{2}{|c|}{$1000 \mathrm{~m} \times 1000 \mathrm{~m}$} \\
\hline Number of nodes & \multicolumn{2}{|c|}{25 (low density), 50 (moderate density) and 75 (high density) } \\
\hline Transmission Range & \multicolumn{2}{|c|}{$250 \mathrm{~m}$} \\
\hline Physical Layer & Signal Propagation Model & Two-ray ground reflection model \\
\hline \multirow{3}{*}{ MAC Layer } & \multicolumn{2}{|c|}{ IEEE 802.11 (Bianchi, 2000) } \\
\hline & Link Bandwidth & $2 \mathrm{Mbps}$ \\
\hline & Interface Queue & FIFO-based, size 100 \\
\hline Routing Protocols & \multicolumn{2}{|c|}{ BEMRP, MAODV, NR-MLPBR and R-MLPBR } \\
\hline Broadcast Strategy & \multirow{2}{*}{\multicolumn{2}{|c|}{$\begin{array}{l}\text { Flooding and DMEF } \\
\text { Random Wav Point Model (Bettstetter et al., 2004) }\end{array}$}} \\
\hline \multirow{4}{*}{ Mobility Model } & & \\
\hline & Minimum Node Speed, $\mathrm{m} / \mathrm{s}$ & $0 \mathrm{~m} / \mathrm{s}$ \\
\hline & Maximum Node Speed, $\mathrm{m} / \mathrm{s}$ & Low-10; Medium-30; High-50 \\
\hline & Pause Time & 0 second \\
\hline \multirow{4}{*}{ Traffic Model } & \multicolumn{2}{|c|}{ Constant Bit Rate (CBR), UDP } \\
\hline & Multicast Group Size (\# Receivers) & Small: 2; Medium: 4, 8; High: 12, 24 \\
\hline & Data Packet Size & 512 bytes \\
\hline & Packet Sending Rate & 4 Packets/ second \\
\hline
\end{tabular}

Simulations are conducted with a multicast group size of 2, 4 (small size), 8, 12 (moderate size) and 24 (larger size) receiver nodes. For each group size, we generated 5 lists of receiver nodes and simulations were conducted with each of them. Traffic sources are constant bit rate (CBR). Data packets are 512 bytes in size and the packet sending rate is 4 data packets/second. The multicast session continues until the end of the simulation time, which is 1000 seconds. The node mobility model used is the Random Waypoint model (Bettstetter et al., 2004). The transmission energy and reception energy per hop is set at $1.4 \mathrm{~W}$ and $1 \mathrm{~W}$ respectively. Initial energy at each node is 1000 Joules. Each node periodically broadcasts a beacon message within its neighborhood to make its presence felt to the other nodes in the neighborhood.

4.1 Multicast Extension of the Ad hoc On-demand Distance Vector (MAODV) Routing Protocol: MAODV (Royer and Perkins, 1999) is the multicast extension of the well-known Ad hoc On-demand Distance Vector (AODV) unicast routing protocol (Perkins and Royer, 1999). Here, a receiver node joins the multicast tree through a member node that lies on the minimum-hop path to the source. A potential receiver wishing to join the multicast group broadcasts a Route-Request (RREQ) message. If a node receives the RREQ message and is not part of the multicast tree, the node broadcasts the message in its neighborhood and also establishes the reverse path by storing the state information consisting of the group address, requesting node id and the sender node id in a temporary cache. If a node receiving the RREQ message is a member of the multicast tree and has not seen the RREQ message earlier, the node waits to receive several RREQ messages and sends back a Route-Reply (RREP) message on the shortest path to the receiver. The member node also informs in the RREP message, the number of hops from itself to the source. The potential receiver receives several RREP messages and selects the member node which lies on the shortest path to the source. The receiver node sends a Multicast Activation (MACT) message to the selected member node along the chosen route. The route from the source to receiver is set up when the member node and all the intermediate nodes in the chosen path update their multicast table with state information from the temporary cache. A similar approach can be used in NR-MLPBR and R-MLPBR when a new receiver node wishes to join the multicast group. Tree maintenance in MAODV is based on the expanding ring search (ERS) approach, using the RREQ, RREP and MACT messages. The downstream node of a broken link is responsible for initiating ERS to issue a fresh RREQ for the group. This RREQ contains the hop count of the requesting node from the source and the last known sequence number for that group. It can be replied only by the member nodes whose recorded sequence number is greater than that indicated in the RREQ and whose hop distance to the source is smaller than the value indicated in the RREQ.

4.2 Bandwidth-Efficient Multicast Routing Protocol (BEMRP): According to BEMRP (Ozaki et al., 2001), a newly joining node to the multicast group opts for the nearest forwarding node in the existing tree, rather than choosing a minimum-hop count path from the source of the multicast group. As a result, the number of links in the multicast tree is reduced leading to savings in the network bandwidth. Multicast tree construction is receiver-initiated. When a node wishes to join the multicast group as a receiver, it initiates the flooding of Join control packets targeted towards the nodes that are currently members of the multicast tree. On 
receiving the first Join control packet, the member node waits for a certain time before sending a Reply packet. The member node sends a Reply packet on the path, traversed by the Join control packet, with the minimum number of intermediate forwarding nodes. The newly joining receiver node collects the Reply packets from different member nodes and would send a Reserve packet on that path that has the minimum number of forwarding nodes from the member node to itself. To provide more bandwidth efficiency, the tree maintenance approach in BEMRP is hard-state based, i.e. a member node transmits control packets only after a link breaks. BEMRP uses two schemes to recover from link failures: Broadcast-multicast scheme - the upstream node of the broken link is responsible for finding a new route to the previous downstream node; Local-rejoin scheme - the downstream node of the broken link tries to rejoin the multicast group using a limited flooding of the Join control packets.

4.3 Broadcast Strategy - Flooding: Flooding is a widely-used approach for disseminating a message from one node to all the nodes in a network. In the case of on-demand ad hoc routing protocols, flooding has been also used to discover a path between a pair of nodes in the network, whenever required (Murthy and Manoj, 2004). For a given network density, flooding offers the highest probability for each node in the network to receive one or more copies of the flooded message. We simulate flooding as follows: The initiating source node sets a monotonically increasing value for the Multicast Tree Request Message (MTRM) and broadcasts the message to its complete neighborhood formed by the default maximum transmission range of the node. Each node that receives the MTRM checks if it has received a MTRM with the same or higher sequence number. If so, the received MTRM is simply discarded. Otherwise, the intermediate node inserts its own ID in the Route Record field of the MTRM and broadcasts the message within its complete neighborhood. Each receiver node of the multicast group upon receiving the first MTRM of a broadcast tree discovery process will include their ID in the route record field and rebroadcast that MTRM further. To select a route to reply back to the source, the receiver node collects the MTRMs received from different paths, selects the minimum hop path and sends a Multicast Tree Establishment Message (MTEM) on the selected minimum hop path to the source.

4.4 Broadcast Strategy - DMEF: Meghanathan (2009b) had proposed a density and mobility aware energy-efficient broadcast strategy (called DMEF) to discover long-living stable routes with a reduced energy spent during route discovery. DMEF takes into consideration the number of neighbors of a node (a measure of network density) and node mobility. The average hop count of the routes discovered using DMEF is only at most about $8 \%$ more than that discovered using flooding.

We simulate DMEF as follows for broadcast multicast tree discoveries: The transmission range of a MTRM broadcast is not fixed for every node. A node that is surrounded by more neighbors in the complete neighborhood will broadcast the MTRM only within a smaller neighborhood that would be sufficient enough to pick up the message and forward it to the other nodes in the rest of the network. On the other hand, a node that is surrounded by fewer neighbors in the complete neighborhood will broadcast the MTRM to a larger neighborhood (but still contained within the complete neighborhood) so that a majority of the nodes in the complete neighborhood can pick up the message and rebroadcast it further. A node rebroadcasts a MTRM at most once. The density aspect of DMEF thus helps to reduce the unnecessary transmission and reception of broadcast MTRMs and conserves energy. To discover stable trees that exist for a longer time, DMEF takes the following approach: A node that is highly mobile makes itself available only to a smaller neighborhood around itself, whereas a node that is less mobile makes itself available over a larger neighborhood (but still contained within the complete neighborhood). The reasoning is that links involving a slow moving node will exist for a long time. Hence, it is better for a slow moving node to advertise itself to a larger neighborhood so that the links (involving this node) that are part of the routes discovered will exist for a longer time. On the other hand, a fast moving node will have links of relatively longer lifetime with neighbors that are closer to it. Hence, it is worth to let a fast moving node advertise only to its nearby neighbors. The rest of the broadcast process is similar to flooding. The receiver node upon receiving the first MTRM will include its identification field in the MTRM and rebroadcast it further depending on its current perceived neighborhood density and own mobility. To select a route to reply back to the source, the receiver node collects the MTRMs received from different paths, selects the minimum hop path and sends a Multicast Tree Establishment Message (MTEM) on the selected minimum hop path to the source.

4.5 Performance Metrics: The performance metrics studied through this simulation are the following:

- Number of Links per Tree: This is the time averaged number of links in the multicast trees discovered and computed over the entire multicast session. The notion of "time-average" is explained as follows: Let there be multicast trees T1, T2, T3 with 5, 8 and 6 links used for time 12, 6 and 15 seconds respectively, then the time averaged number of links in the multicast trees is given by $(5 * 12+8 * 6+6 * 15) /(12+6+15)=6$ and not merely 6.33 , which is the average of 5,8 and 6 .

- Hop Count per Source-Receiver Path: This is the time averaged hop count of the paths from the source to each receiver of the multicast group and computed over the entire multicast session.

- Time between Successive Broadcast Tree Discoveries: This is the time between two successive broadcast tree discoveries, averaged over the entire multicast session. This metric is a measure of the lifetime of the multicast trees discovered and also the effectiveness of the path prediction approach followed in NR-MLPBR and R-MLPBR.

- Energy Consumed per Node: This is the sum of the energy consumed at a node due to the transfer of data packets as part of the multicast session, broadcast tree discoveries as well as the periodic broadcast and exchange of beacons in the neighborhood. 
- Energy Throughput: This is the average of the ratio of the number of data packets reaching the destination to the sum of the energy spent across all the nodes in the network.

- Energy Consumed per Tree Discovery: This is the average of the total energy consumed for the global broadcast based tree discovery attempts. This includes the sum of the energy consumed to transmit (broadcast) the MTRM packets to the nodes in the neighborhood and to receive the MTRM packet sent by each node in the neighborhood, summed over all the nodes. It also includes the energy consumed to transmit the MTEM packet from each receiver to the source of the multicast session.

\section{Simulation Results}

The performance results for each metric displayed in Figures 12 through 22 are an average of the results obtained from simulations conducted with 5 sets of multicast groups and 5 sets of mobility profiles for each group size, node velocity and network density values. The multicast source in each case was selected randomly among the nodes in the network and the source is not part of the multicast group. The nodes that are part of the multicast group are merely the receivers.

5.1 Number of Links per Multicast Tree: The number of links per multicast tree (refer Figures 12 and 13) is a measure of the efficiency of the multicast routing protocol in reducing the number of link transmissions during the transfer of the multicast data from the source to the receivers of the multicast group. The smaller is the number of links in the tree, the larger the link transmission efficiency of the multicast routing protocol. If fewer links are part of the tree, then the chances of multiple transmissions in the network increase and this increases the efficiency of link usage and the network bandwidth. Naturally, the BEMRP protocol, which has been purely designed to yield bandwidth-efficient multicast trees, discovers trees that have a reduced number of links for all the operating scenarios. This leads to larger hop count per source-receiver paths for BEMRP as observed in Figures 14 and 15. R-MLPBR, which has been designed to choose the predicted paths with the minimum number of non-receiver nodes, manages to significantly reduce the number of links vis-à-vis the MAODV and NR-MLPBR protocols. R-MLPBR attempts to minimize the number of links in the multicast tree without yielding to a higher hop count per source-receiver path. But, the tradeoff between the link efficiency and the hop count per source-receiver path continues to exist and it cannot be nullified. In other words, R-MLPBR cannot discover trees that have minimum number of links as well as the minimum hop count per sourcereceiver path. Nevertheless, R-MLPBR is the first multicast routing protocol that yields trees with the reduced number of links and at the same time, with a reduced hop count (close to the minimum) per source-receiver path.

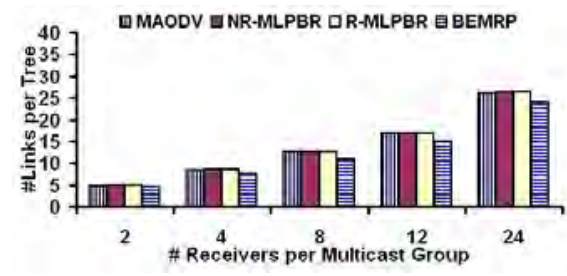

Figure 12.1. 25 nodes, $10 \mathrm{~m} / \mathrm{s}$

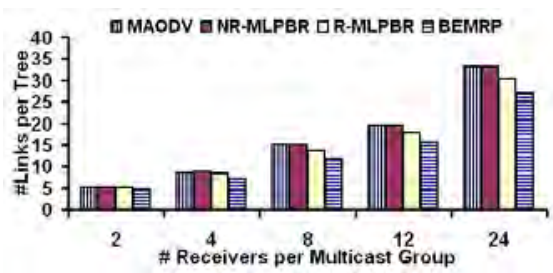

Figure 12.4. 50 nodes, $10 \mathrm{~m} / \mathrm{s}$

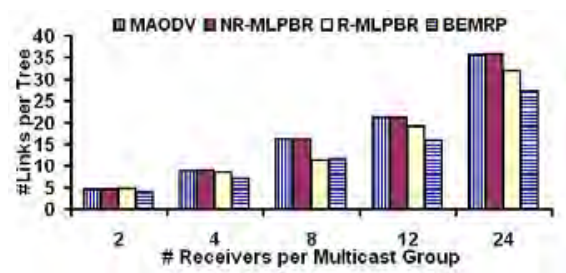

Figure 12.7. 75 nodes, $10 \mathrm{~m} / \mathrm{s}$

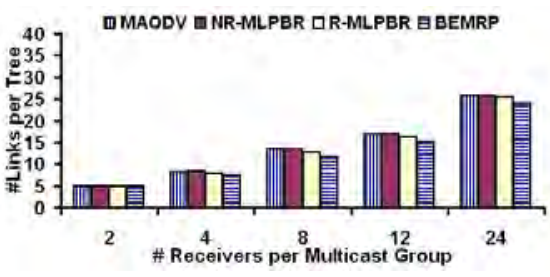

Figure 12.2. 25 nodes, $30 \mathrm{~m} / \mathrm{s}$

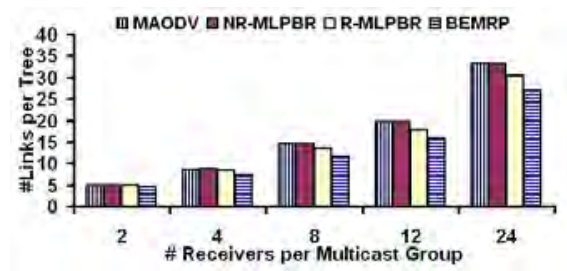

Figure 12.5. 50 nodes, $30 \mathrm{~m} / \mathrm{s}$

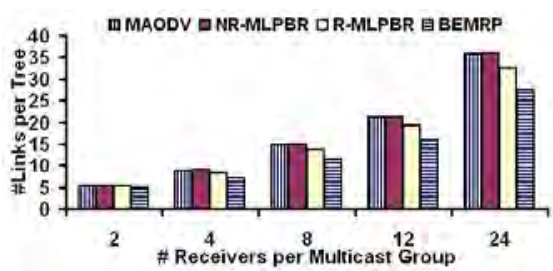

Figure 12.8. 75 nodes, $30 \mathrm{~m} / \mathrm{s}$

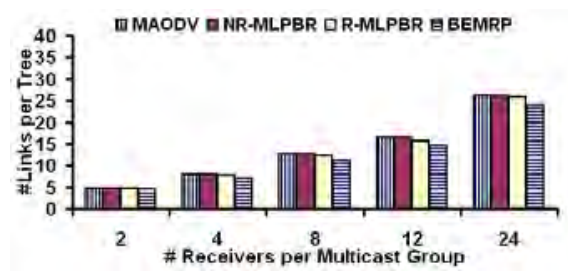

Figure 12.3. 25 nodes, $50 \mathrm{~m} / \mathrm{s}$

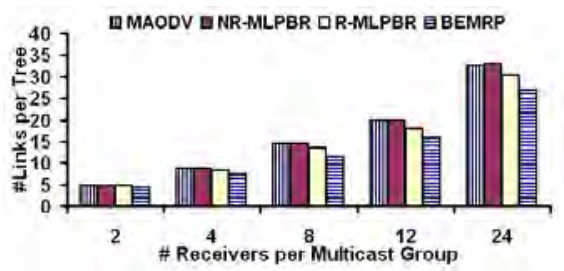

Figure 12.6. 50 nodes, $50 \mathrm{~m} / \mathrm{s}$

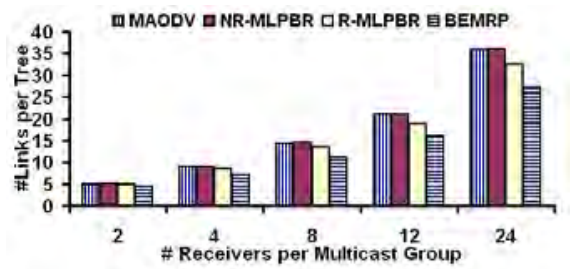

Figure 12.9. 75 nodes, $50 \mathrm{~m} / \mathrm{s}$

Figure 12. Average Number of Links per Multicast Tree (Tree Discovery Procedure: Flooding) 
With flooding (refer Figure 12), for a given network density and multicast group size, as we increase the node velocity, we do not see any appreciable variation in the number of links per tree for each of the multicast routing protocols studied. On the other hand, with DMEF (refer Figure 13), for each of the multicast routing protocols, as the maximum node velocity is increased from $10 \mathrm{~m} / \mathrm{s}$ to $30 \mathrm{~m} / \mathrm{s}$ and then to $50 \mathrm{~m} / \mathrm{s}$, the number of links per multicast tree increases as large as up to $24 \%$. This shows that DMEF can yield multicast trees with reduced number of links in low node mobility, especially for multicast groups of small and moderate sizes. With flooding (refer Figure 12), for a given multicast group size, as the network density increases, the number of links per tree for MAODV and NR-MLPBR is increasingly more than that incurred with BEMRP. As the network density increases, BEMRP attempts to reduce the number of links per tree by incorporating links that can be shared by multiple receivers on the paths towards the source. On the other hand, both MAODV and NR-MLPBR attempt to choose minimum hop paths between the source and any receiver and hence exploit the increase in network density to discover minimum hop paths, but at the cost of the link efficiency. On the other hand, like BEMRP, R-MLPBR also attempts to reduce the number of links per tree as we increase the network density, but at a lower rate. Similar performance results can also be attributed to the impact of network density on the performance of the routing protocols when DMEF (refer Figure 13) is used as the broadcast tree discovery strategy. With flooding (refer Figure 12), for a given network density and node mobility, as the multicast group size increases, the number of links per tree for MAODV and NR-MLPBR is larger and increases significantly compared to BEMRP and R-MLPBR. R-MLPBR is relatively more scalable, similar to BEMRP, with increase in the multicast group size. Similar performance results can also be attributed to the impact of multicast group size on the performance of the routing protocols when DMEF (refer Figure 13) is used as the broadcast tree discovery strategy.

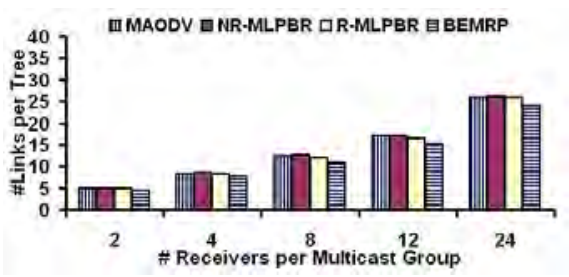

Figure 13.1. 25 nodes, $10 \mathrm{~m} / \mathrm{s}$

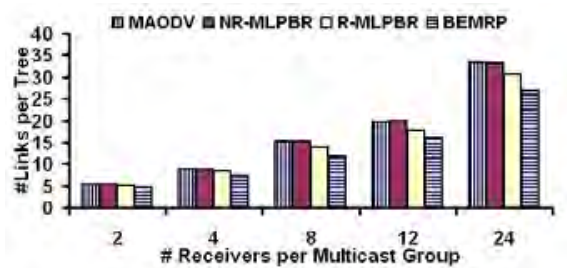

Figure 13.4. 50 nodes, $10 \mathrm{~m} / \mathrm{s}$

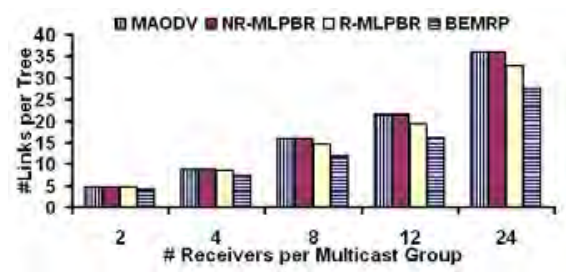

Figure 13.7. 75 nodes, $10 \mathrm{~m} / \mathrm{s}$

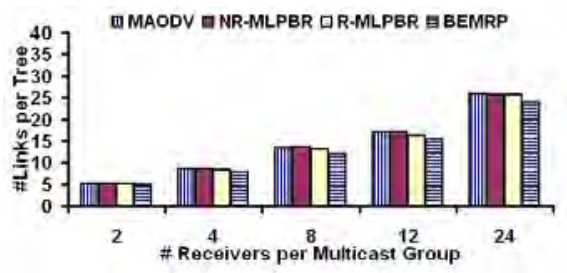

Figure 13.2. 25 nodes, $30 \mathrm{~m} / \mathrm{s}$

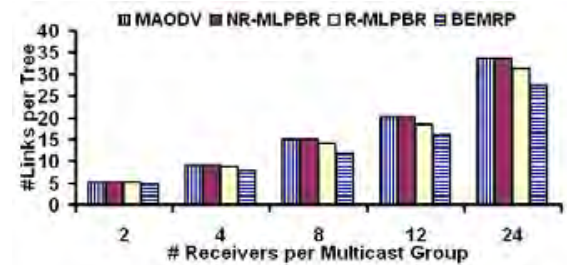

Figure 13.5. 50 nodes, $30 \mathrm{~m} / \mathrm{s}$

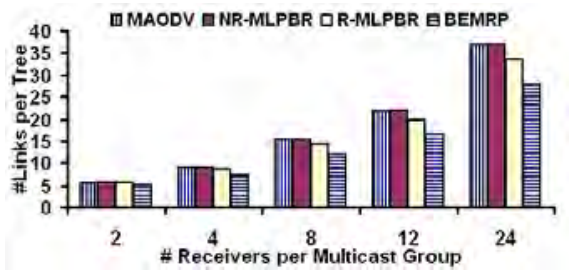

Figure 13.8. 75 nodes, $30 \mathrm{~m} / \mathrm{s}$

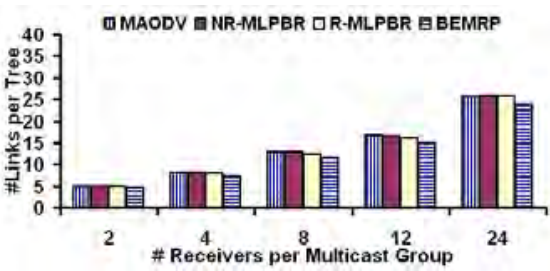

Figure 13.3. 25 nodes, $50 \mathrm{~m} / \mathrm{s}$

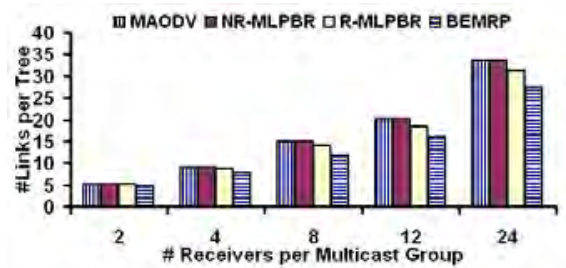

Figure 13.6. 50 nodes, $50 \mathrm{~m} / \mathrm{s}$

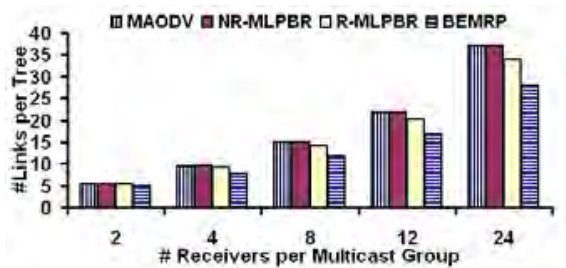

Figure 13.9. 75 nodes, $50 \mathrm{~m} / \mathrm{s}$

Figure 13. Average Number of Links per Multicast Tree (Tree Discovery Procedure: DMEF)

5.2 Average Hop Count per Source-Receiver Path: All the three multicast routing protocols - MAODV, NR-MLPBR and RMLPBR, incur almost the same average hop count per source-receiver and it is considerably lower than that incurred for BEMRP. The hop count per source-receiver path is an important metric and it is often indicative of the end-to-end delay per multicast packet from the source to a specific receiver. BEMRP incurs a significantly larger hop count per source-receiver path and this can be attributed to the nature of this multicast routing protocol to look for trees with a reduced number of links. When multiple receiver nodes have to be connected to the source through a reduced set of links, the hop count per source-receiver path is bound to increase. In Performance Figures 14 and 15, we can see a significant increase in the hop count per source-receiver path as we increase the multicast group size. In the case of flooding, the hop count per source-receiver path for BEMRP can be as large as $41 \%$, 57\% and 59\% more than that of the hop count per source-receiver path incurred for the other three multicast routing protocols. In the case of DMEF, the hop count per source-receiver path for BEMRP can be as large as 36\%, 49\% and 53\% more 
than that of the hop count per source-receiver path incurred for the other three multicast routing protocols. The increase in the hop count per source-receiver path for BEMRP with DMEF is thus slightly lower than that obtained under flooding.

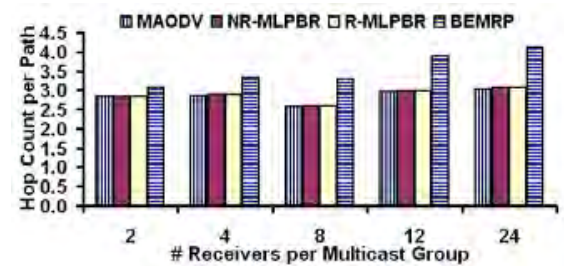

Figure 14.1. 25 nodes, $10 \mathrm{~m} / \mathrm{s}$

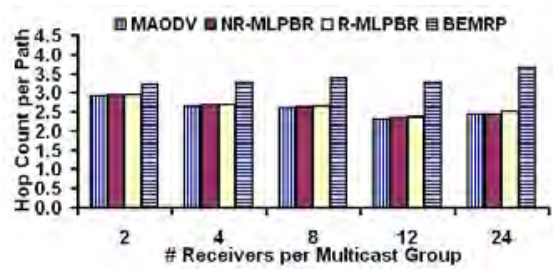

Figure 14.4. 50 nodes, $10 \mathrm{~m} / \mathrm{s}$

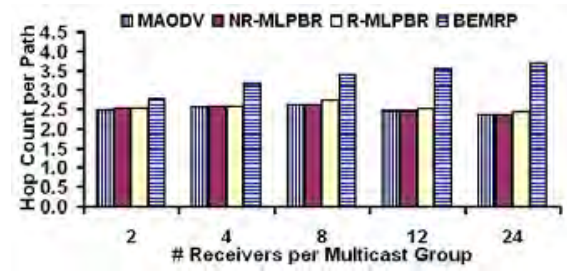

Figure 14.7. 75 nodes, $10 \mathrm{~m} / \mathrm{s}$

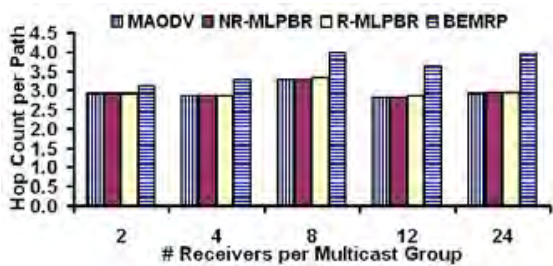

Figure 14.2. 25 nodes, $30 \mathrm{~m} / \mathrm{s}$

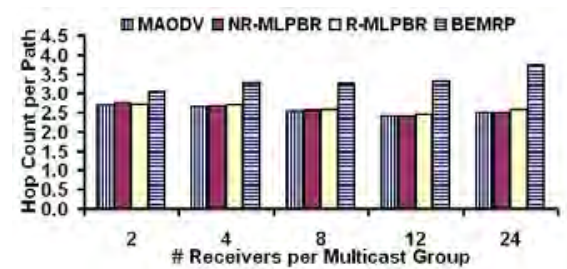

Figure 14.5. 50 nodes, $30 \mathrm{~m} / \mathrm{s}$

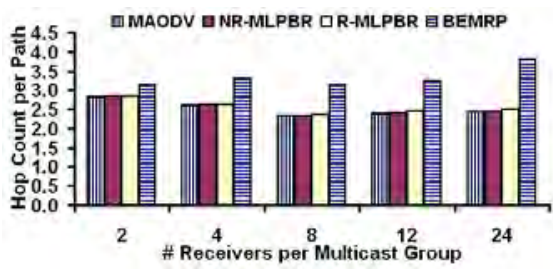

Figure 14.8. 75 nodes, $30 \mathrm{~m} / \mathrm{s}$

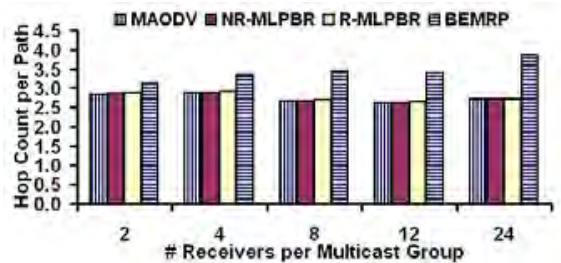

Figure 14.3. 25 nodes, $50 \mathrm{~m} / \mathrm{s}$

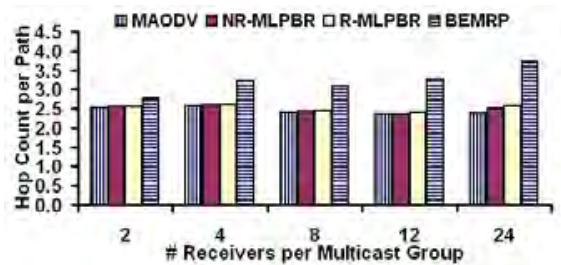

Figure 14.6. 50 nodes, $50 \mathrm{~m} / \mathrm{s}$

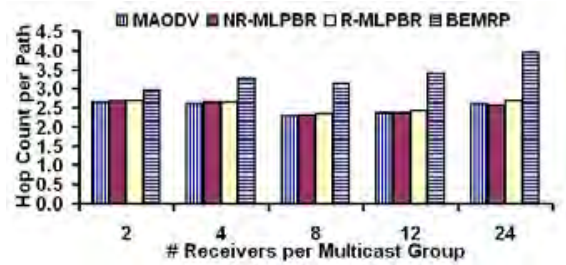

Figure 14.9. 75 nodes, $50 \mathrm{~m} / \mathrm{s}$

Figure 14. Average Hop Count per Source-Receiver Path (Tree Discovery Procedure: Flooding)

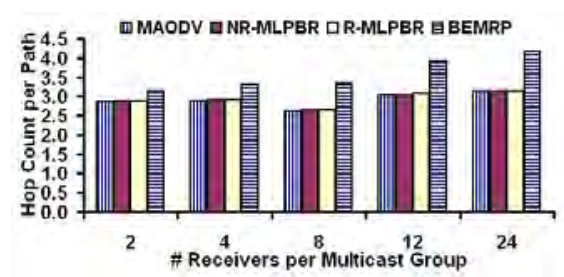

Figure 15.1. 25 nodes, $10 \mathrm{~m} / \mathrm{s}$

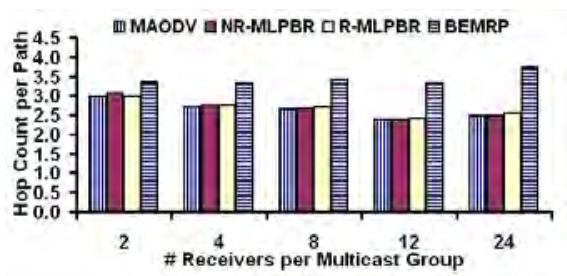

Figure 15.4. 50 nodes, $10 \mathrm{~m} / \mathrm{s}$

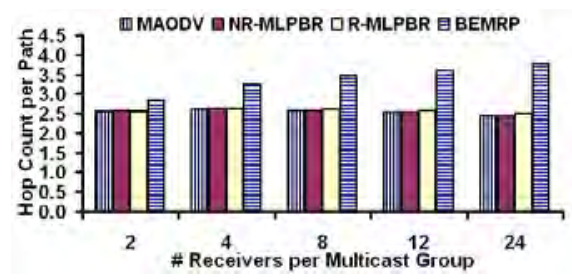

Figure 15.7. 75 nodes, $10 \mathrm{~m} / \mathrm{s}$

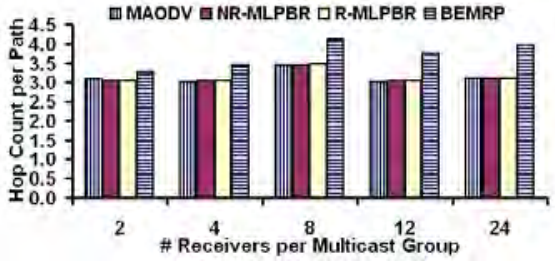

Figure 15.2. 25 nodes, $30 \mathrm{~m} / \mathrm{s}$

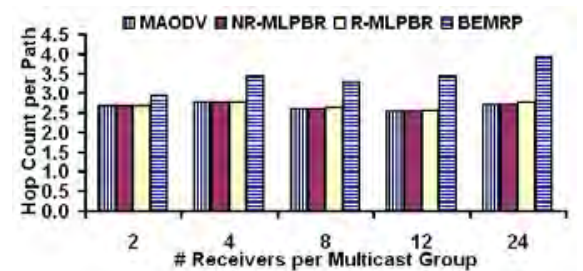

Figure 15.5. 50 nodes, $30 \mathrm{~m} / \mathrm{s}$

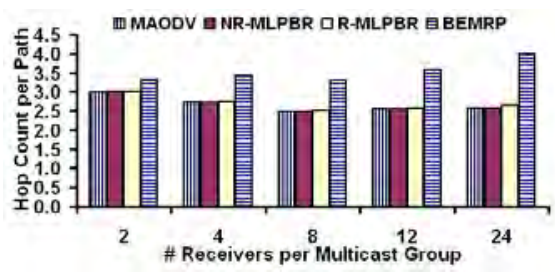

Figure 15.8. 75 nodes, $30 \mathrm{~m} / \mathrm{s}$

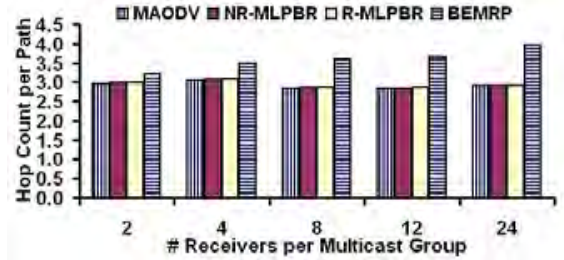

Figure 15.3. 25 nodes, $50 \mathrm{~m} / \mathrm{s}$

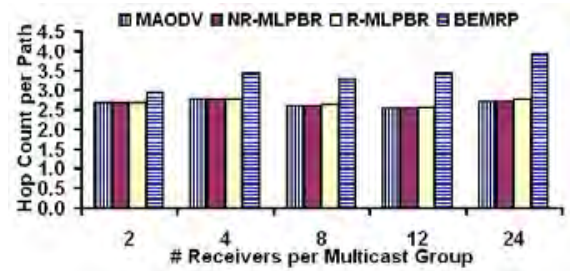

Figure 15.6. 50 nodes, $50 \mathrm{~m} / \mathrm{s}$

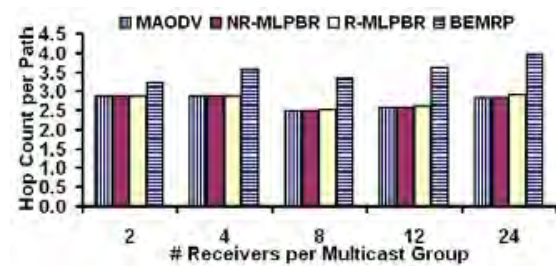

Figure 15.9. 75 nodes, $50 \mathrm{~m} / \mathrm{s}$

Figure 15. Average Hop Count per Source-Receiver Path (Tree Discovery Procedure: DMEF) 
With flooding (refer Figure 14), for a given network density and group size, as we increase node velocity, we do not see any appreciable variation in the hop count per source-receiver path for each of the multicast routing protocols studied. On the other hand, with DMEF (refer Figure 15), for each of the multicast routing protocols, as the maximum node velocity is increased from $10 \mathrm{~m} / \mathrm{s}$ to $30 \mathrm{~m} / \mathrm{s}$ and then to $50 \mathrm{~m} / \mathrm{s}$, the number of links per multicast tree increases as large as up to $17 \%$. This shows that DMEF can yield multicast trees with reduced number of links in low node mobility, especially for multicast groups of small and moderate sizes. Under both flooding and DMEF, for MAODV, NR-MLPBR and R-MLPBR, with increase in network density, the hop count per source-receiver path decreases (as large as 20\%) significantly more than that of BEMRP (limited to only at most $10 \%$ ). For a given network density and node mobility, as we increase the multicast group size from 2 to 24, under both flooding and DMEF, the difference in the hop count per source-receiver path between BEMRP and the other three multicast routing protocols increases from $10 \%$ to $59 \%$.

5.3 Average Time between Successive Broadcast Tree Discoveries: The time between successive broadcast tree discoveries is a measure of the stability of the multicast trees and the effectiveness of the location prediction and path prediction approach of the two multicast extensions of LPBR. For a given condition of node density and node mobility, both NR-MLPBR and R-MLPBR incur relatively larger time between successive broadcast tree discoveries for smaller and medium sized multicast groups. MAODV tends to be more unstable as the multicast group size is increased, owing to the minimum hop nature of the paths discovered and absence of any path prediction approach. For larger multicast groups, BEMRP tends to perform better by virtue of its tendency to strictly minimize only the number of links in the tree. On the other hand, NR-MLPBR attempts to reduce the hop count per source-receiver path and ends up choosing predicted paths that increase the number of links in the tree, quickly leading to the failure of the tree. For a given level of node mobility and network density, MAODV trees become highly unstable as the multicast group size increases. For multicast groups of size 2 and 4, the time between successive broadcast tree discoveries for NR-MLPBR and R-MLPBR is greater than that obtained for BEMRP, especially in networks of low and moderate network density. For larger multicast group sizes, when we employ flooding, BEMRP tends to incur larger time between successive broadcast tree discoveries compared to NR-MLPBR and R-MLPBR. On the other hand, when we employ DMEF, R-MLPBR tends to incur larger time between successive broadcast tree discoveries compared to BEMRP, even for larger group sizes.

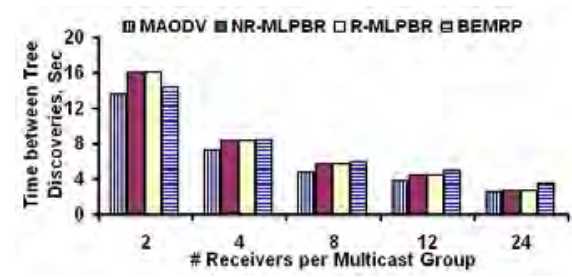

Figure 16.1. 25 nodes, $10 \mathrm{~m} / \mathrm{s}$

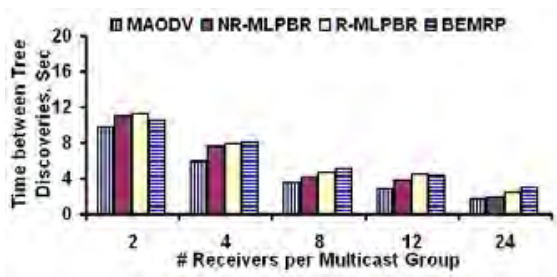

Figure 16.4. 50 nodes, $10 \mathrm{~m} / \mathrm{s}$

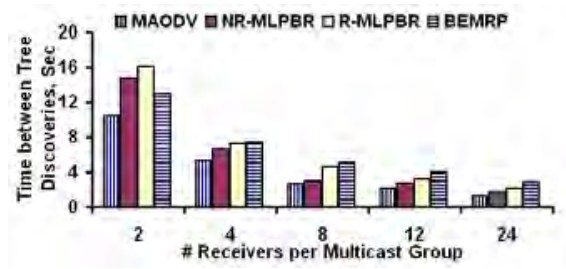

Figure 16.7. 75 nodes, $10 \mathrm{~m} / \mathrm{s}$

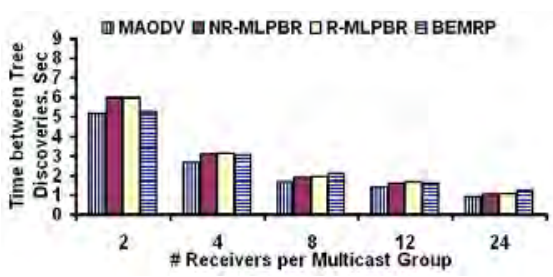

Figure 16.2. 25 nodes, $30 \mathrm{~m} / \mathrm{s}$

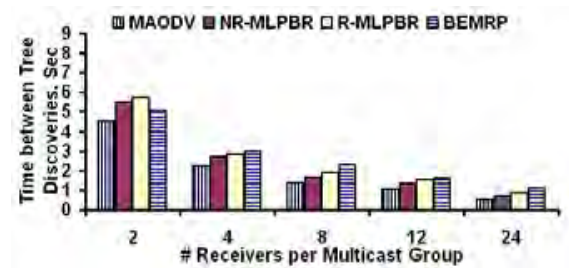

Figure 16.5. 50 nodes, $30 \mathrm{~m} / \mathrm{s}$

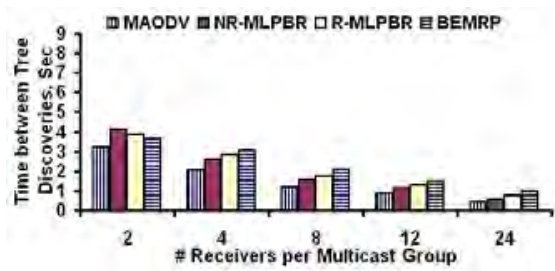

Figure 16.8. 75 nodes, $30 \mathrm{~m} / \mathrm{s}$

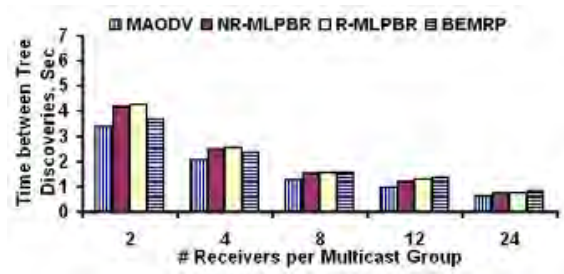

Figure 16.3. 25 nodes, $50 \mathrm{~m} / \mathrm{s}$

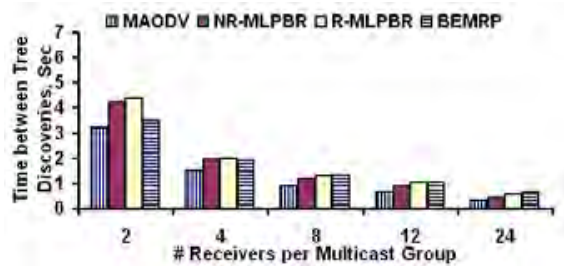

Figure 16.6. 50 nodes, $50 \mathrm{~m} / \mathrm{s}$

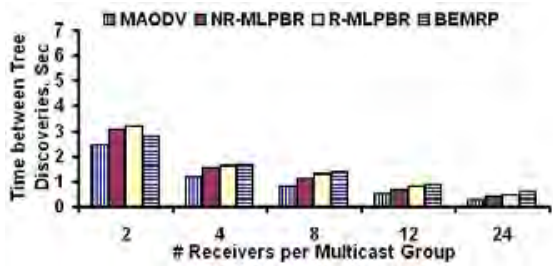

Figure 16.9. 75 nodes, $50 \mathrm{~m} / \mathrm{s}$

Figure 16. Average Time between Successive Tree Discoveries (Tree Discovery Procedure: Flooding)

For a given multicast group size, network density and multicast routing protocol and node mobility, the time between successive route discoveries while using DMEF (refer Figure 17) is at least 10-15\% more than that obtained due to flooding (refer Figure 16). As we increase the maximum node velocity from $10 \mathrm{~m} / \mathrm{s}$ to $30 \mathrm{~m} / \mathrm{s}$ and $50 \mathrm{~m} / \mathrm{s}$, the decrease in the time between successive route discoveries is more when the multicast routing protocols discover the tree using flooding (decrease to $21-28 \%$ of the value at 10 
$\mathrm{m} / \mathrm{s}$ ) compared to DMEF (decrease to $34-38 \%$ of the value at $10 \mathrm{~m} / \mathrm{s}$ ). When flooding is used as the underlying tree discovery strategy, for a given multicast group size and level of node mobility, as the network density increases, the time between successive broadcast tree discoveries decreases. This is mainly observed for the minimum-hop based multicast protocols (especially MAODV and NR-MLPBR) which incur a reduced hop count per source-receiver path as we increase the network density. But, such minimum hop paths obtained in moderate and high-density networks are relatively less stable than those obtained in low-density networks. When DMEF is used as the underlying tree discovery strategy, the larger number of nodes in the neighborhood is taken into account by DMEF to discover stable routes and there is no appreciable difference in the time between successive tree discoveries. With increase in network density, the maximum decrease in the time between successive tree discoveries with DMEF is $13 \%$. For a given network density and node mobility, for each multicast routing protocol, the time between successive tree discoveries decreases with increase in multicast group size - with flooding the difference in the time between successive tree discoveries for MAODV and NR-MLPBR compared with R-MLPBR and BEMRP is as large as $40 \%$ whereas, the difference is only as large as $25 \%$ when DMEF is used.

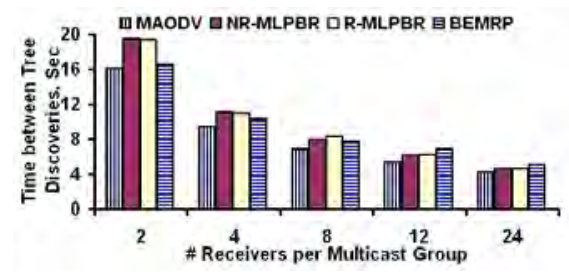

Figure 17.1. 25 nodes, $10 \mathrm{~m} / \mathrm{s}$

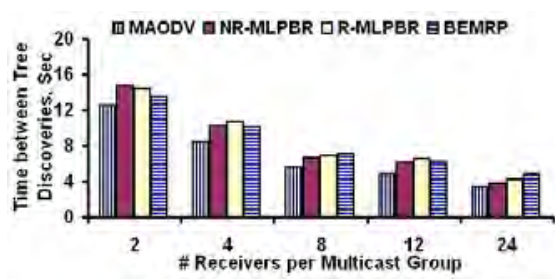

Figure 17.4. 50 nodes, $10 \mathrm{~m} / \mathrm{s}$

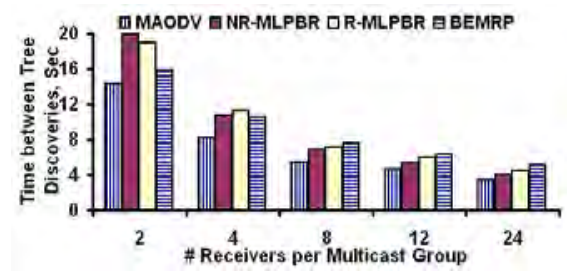

Figure 17.7. 75 nodes, $10 \mathrm{~m} / \mathrm{s}$

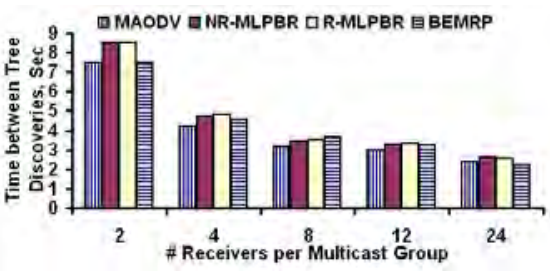

Figure 17.2. 25 nodes, $30 \mathrm{~m} / \mathrm{s}$

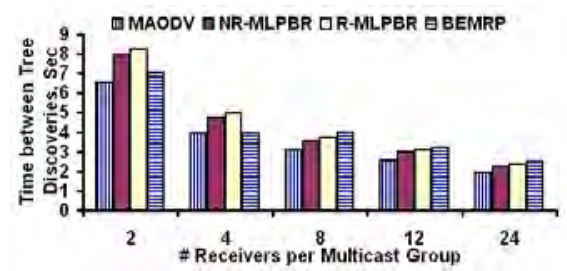

Figure 17.5. 50 nodes, $30 \mathrm{~m} / \mathrm{s}$

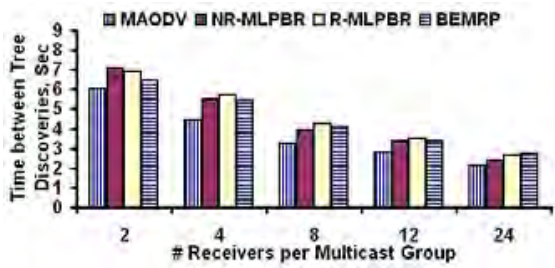

Figure 17.8. 75 nodes, $30 \mathrm{~m} / \mathrm{s}$

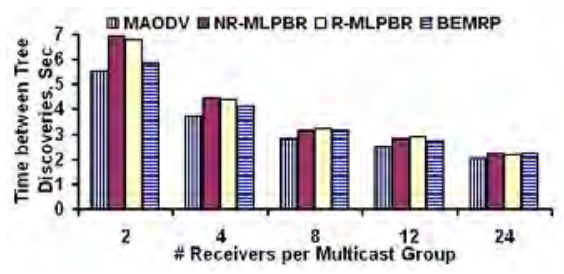

Figure 17.3. 25 nodes, $50 \mathrm{~m} / \mathrm{s}$

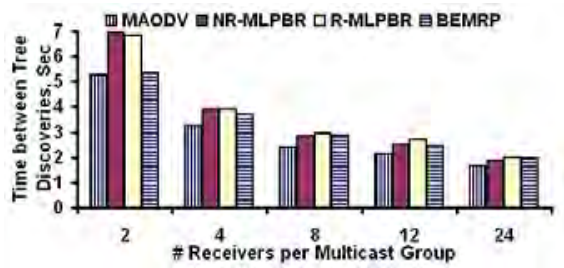

Figure 17.6. 50 nodes, $50 \mathrm{~m} / \mathrm{s}$

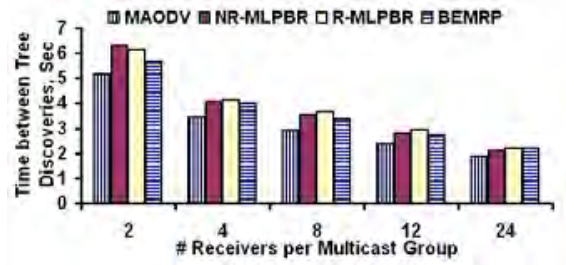

Figure 17.9. 75 nodes, $50 \mathrm{~m} / \mathrm{s}$

Figure 17. Average Time between Successive Tree Discoveries (Tree Discovery Procedure: DMEF)

5.4 Average Energy Consumed per Node: Energy consumption in multicast routing is directly proportional to the number of links in the tree. Larger the number of links, more the transmissions and more will be the energy consumption in the network and viceversa. The simulation results in Figures 18 and 19 clearly illustrate this. BEMRP incurs the least energy consumption per node and MAODV incurs the largest energy consumption per node. The energy consumed per node for the two multicast extensions is in between these two extremes. The energy consumed per node for R-MLPBR is less than that of NR-MLPBR as the former also attempts to simultaneously reduce the number of links as well as the hop count per source-receiver path. The energy consumption per node increases as the multicast group size increases. For a given multicast group size and multicast routing protocol, the energy consumed per node increases with increase in network density as well as with increase in node mobility.

With flooding as the tree discovery strategy (refer Figure 18), for a given multicast group size, network density and multicast routing protocol, the energy consumed per node at maximal node velocity of $30 \mathrm{~m} / \mathrm{s}$ and $50 \mathrm{~m} / \mathrm{s} \mathrm{can} \mathrm{grow} \mathrm{as} \mathrm{large} \mathrm{as} 10-40 \%$ of that obtained at maximal node velocity of $10 \mathrm{~m} / \mathrm{s}$. On the other hand, with DMEF as the tree discovery strategy (refer Figure 19), for a given multicast group size, network density and multicast routing protocol, the energy consumed per node at maximal node velocity of $30 \mathrm{~m} / \mathrm{s}$ and $50 \mathrm{~m} / \mathrm{s}$ can grow as large as $5-20 \%$ of that obtained at maximal node velocity of $10 \mathrm{~m} / \mathrm{s}$. This indicates the effectiveness of DMEF vis-à-vis flooding in reducing the energy consumed per node. DMEF discovers relatively more stable trees by involving only slow moving nodes in the tree. As a result, the multicast trees exist for a longer time and incur less energy for tree discoveries. Under both flooding and DMEF, BEMRP and MAODV incur the largest increase in energy consumed per node with increase in node mobility. NR-MLPBR and R-MLPBR incur a relatively lower increase in the energy consumed per node 
with increase in node mobility. This can be attributed to the tendency of these multicast routing protocols to reduce the number of broadcast tree discoveries using effective tree prediction.

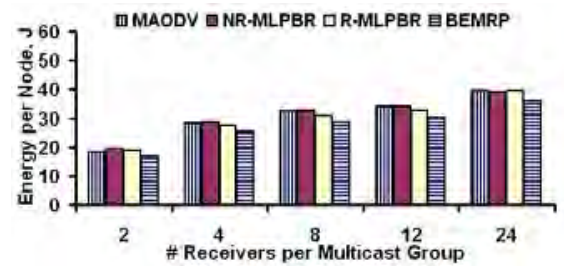

Figure 18.1. 25 nodes, $10 \mathrm{~m} / \mathrm{s}$

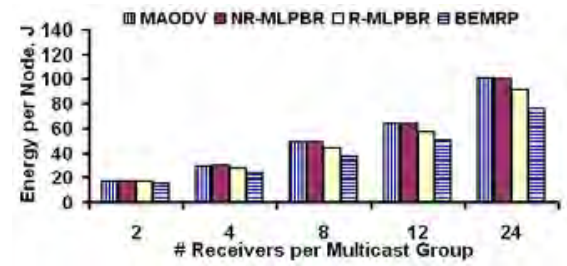

Figure 18.4. 50 nodes, $10 \mathrm{~m} / \mathrm{s}$

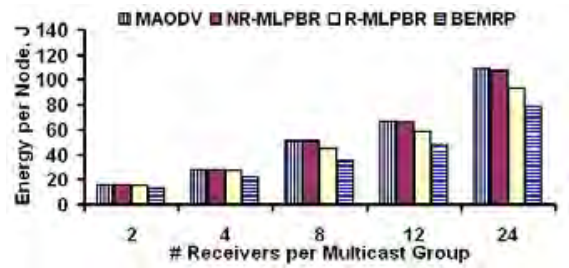

Figure 18.7. 75 nodes, $10 \mathrm{~m} / \mathrm{s}$

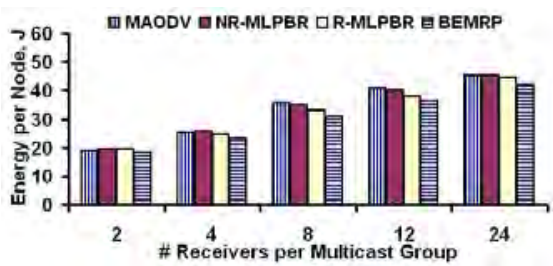

Figure 18.2. 25 nodes, $30 \mathrm{~m} / \mathrm{s}$

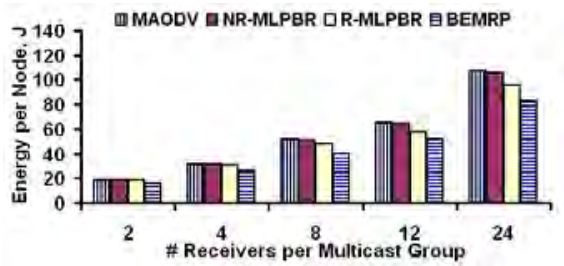

Figure 18.5. 50 nodes, $30 \mathrm{~m} / \mathrm{s}$

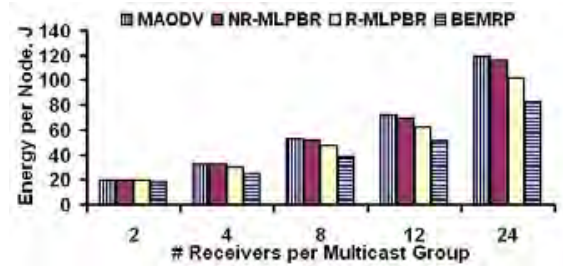

Figure 18.8. 75 nodes, $30 \mathrm{~m} / \mathrm{s}$

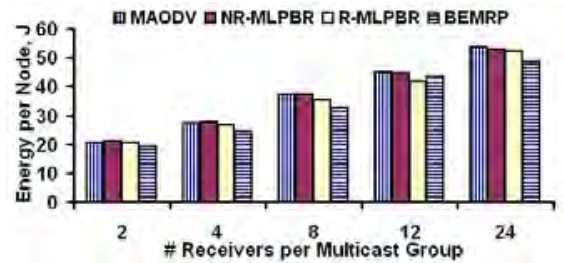

Figure 18.3. 25 nodes, $50 \mathrm{~m} / \mathrm{s}$

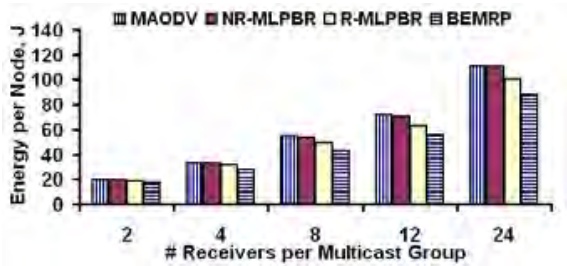

Figure 18.6. 50 nodes, $50 \mathrm{~m} / \mathrm{s}$

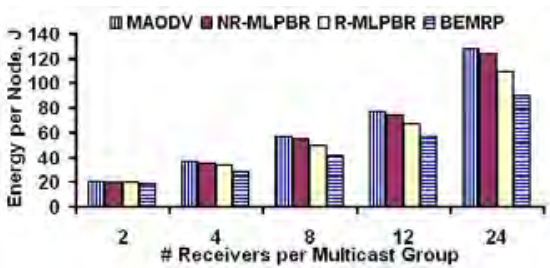

Figure 18.9. 75 nodes, $50 \mathrm{~m} / \mathrm{s}$

Figure 18. Average Energy Consumed per Node (Tree Discovery Procedure: Flooding)

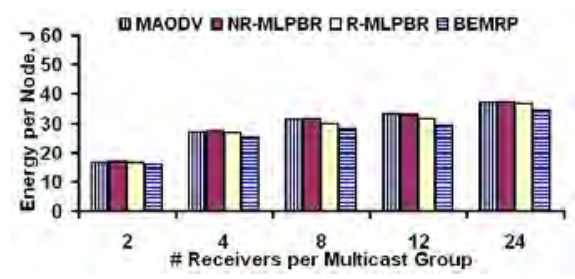

Figure 19.1. 25 nodes, $10 \mathrm{~m} / \mathrm{s}$

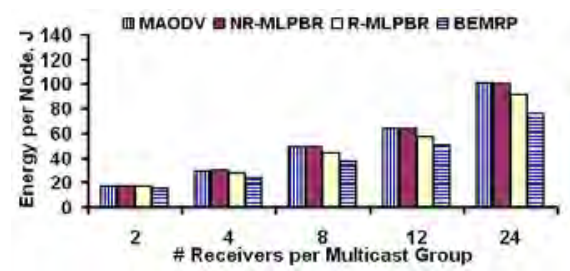

Figure 19.4. 50 nodes, $10 \mathrm{~m} / \mathrm{s}$

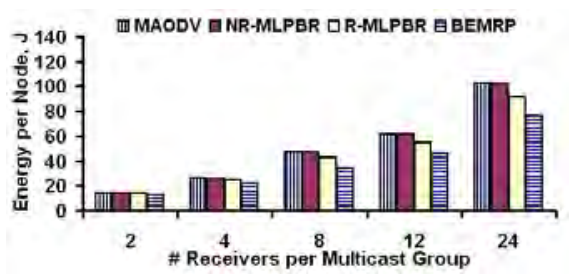

Figure 19.7. 75 nodes, $10 \mathrm{~m} / \mathrm{s}$

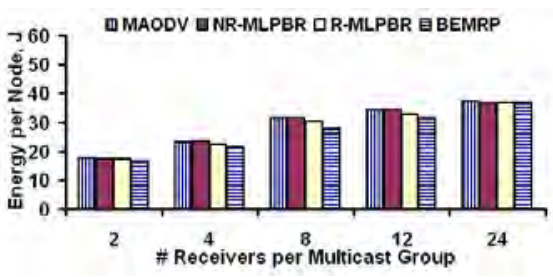

Figure 19.2. 25 nodes, $30 \mathrm{~m} / \mathrm{s}$

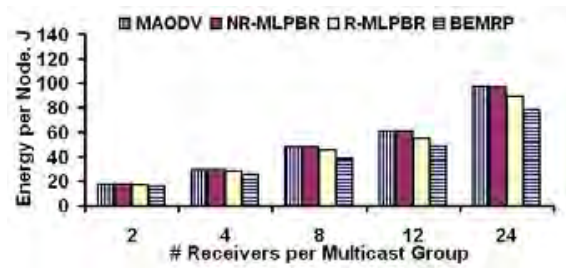

Figure 19.5. 50 nodes, $30 \mathrm{~m} / \mathrm{s}$

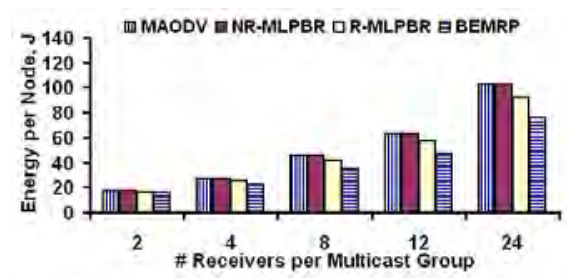

Figure 19.8. 75 nodes, $30 \mathrm{~m} / \mathrm{s}$

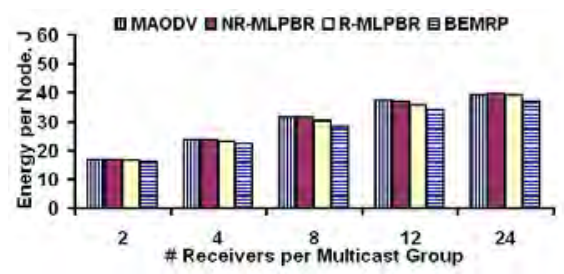

Figure 19.3. 25 nodes, $50 \mathrm{~m} / \mathrm{s}$

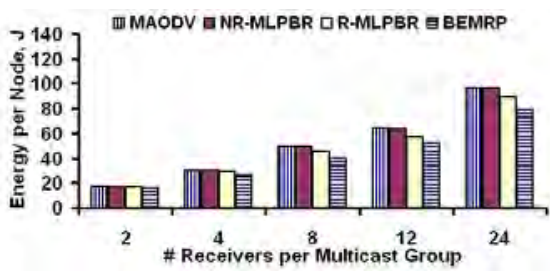

Figure 19.6. 50 nodes, $50 \mathrm{~m} / \mathrm{s}$

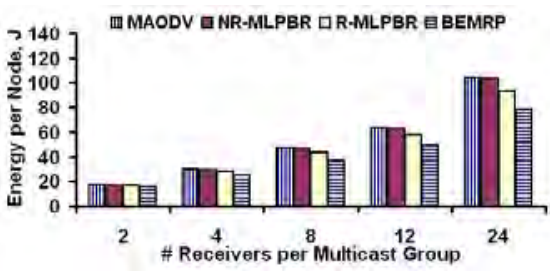

Figure 19.9. 75 nodes, $50 \mathrm{~m} / \mathrm{s}$

Figure 19. Average Energy Consumed per Node (Tree Discovery Procedure: DMEF) 
We observe that for a given multicast routing protocol, for a given network density, the energy consumed per node due to flooding can be as large as 5\%-16\%, 12\%-23\% and 22\%-37\% more than that incurred using DMEF in the presence of low, medium and high node mobility respectively. For both flooding and DMEF, for multicast groups of size 2 and 4, we observe that with increase in network density from 25 to 50 nodes and from 25 to 75 nodes, the energy consumed per node decreases. This can be attributed to the smaller group size, leading to the effective sharing of the data forwarding load among all the nodes in the network. For larger group sizes, all the nodes in the network end up spending more energy (due to transmission/reception or at least receiving the packets in the neighborhood). As a result, for multicast group sizes of 8, 12 and 24, as we increase the network density from 25 nodes to 50 and 75 nodes, the energy consumed per node for each of the multicast routing protocol increases significantly. MAODV and NR-MLPBR incur a relatively larger energy consumed per node at high network densities due to the nature of these multicast routing protocols to discover trees with minimum hop count. R-MLPBR and BEMRP discover trees with reduced number of links and hence incur relatively lower energy consumed per node at high network density. For both flooding and DMEF, for a given network density, node mobility and multicast routing protocol, as we increase the multicast group size from 2 to 24, the increase in the energy consumed per node is below linear. Hence, all the four multicast routing protocols are scalable with respect to the increase in multicast group size.

5.5 Energy Throughput: For each of the multicast routing protocols and for a given network density and node mobility, the energy throughput decreases with increase in the multicast group size. This can be attributed to the need to spend more energy to deliver a given multicast packet to more receivers vis-à-vis few receivers. For a given network density and multicast group size, the energy throughput of a multicast routing protocol decreases slightly as the node velocity is increased from low to moderate and high. For a given multicast group size and node mobility, the energy throughput of a multicast routing protocol decreases with increase in network density. This can be attributed to the involvement of several nodes (for larger network density) in distributing the offered traffic load to the multicast group. For a given simulation condition, the energy throughput of BEMRP is slightly larger than that of the other multicast routing protocols. This can be attributed to the lower energy consumed per node (and less number of links) for BEMRP.

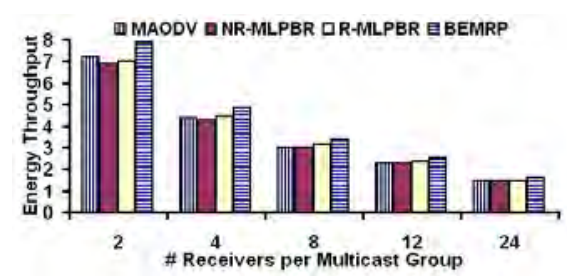

Figure 20.1. 25 nodes, $10 \mathrm{~m} / \mathrm{s}$

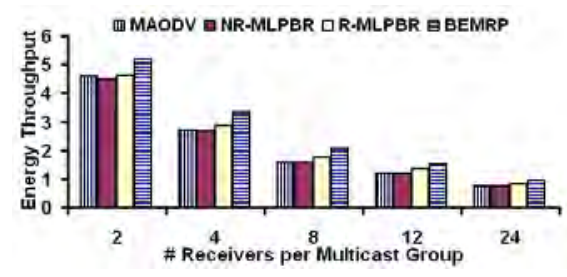

Figure 20.4. 50 nodes, $10 \mathrm{~m} / \mathrm{s}$

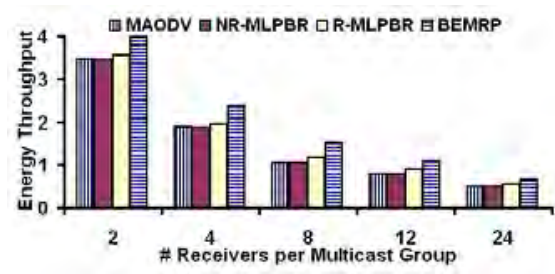

Figure 20.7. 75 nodes, $10 \mathrm{~m} / \mathrm{s}$

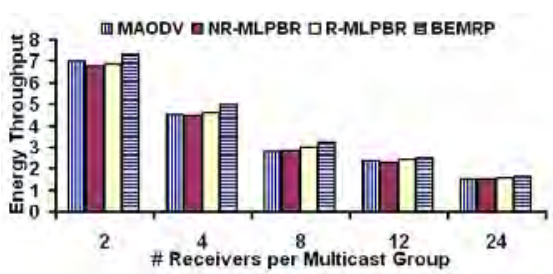

Figure 20.2. 25 nodes, $30 \mathrm{~m} / \mathrm{s}$

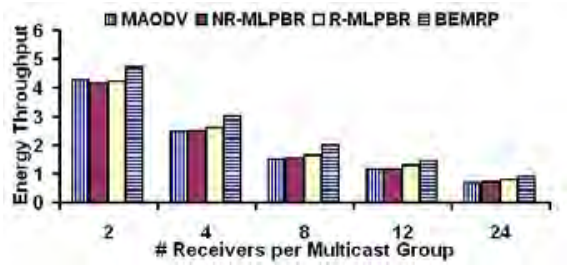

Figure 20.5. 50 nodes, $30 \mathrm{~m} / \mathrm{s}$

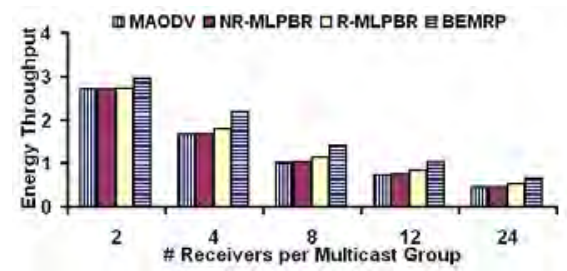

Figure 20.8. 75 nodes, $30 \mathrm{~m} / \mathrm{s}$

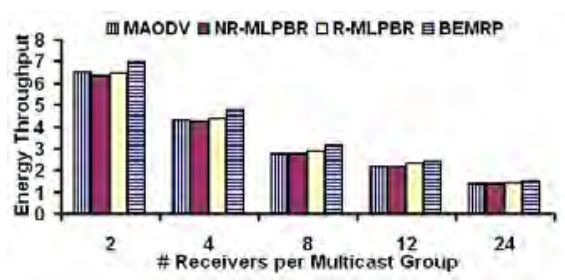

Figure 20.3. 25 nodes, $50 \mathrm{~m} / \mathrm{s}$

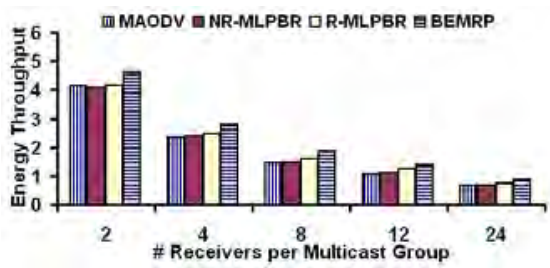

Figure 20.6. 50 nodes, $50 \mathrm{~m} / \mathrm{s}$

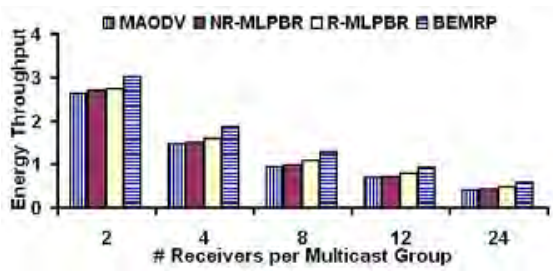

Figure 20.9. 75 nodes, $50 \mathrm{~m} / \mathrm{s}$

Figure 20. Energy Throughput: \# Packets Delivered per Joule (Tree Discovery Procedure: Flooding)

For a given multicast routing protocol, as we increase the node mobility from low to moderate and high, the energy throughput for a multicast routing protocol decreases with increase in network density and the reduction can be as large as $26 \%$ and $17 \%$ for flooding and DMEF respectively. For a given network density, the reduction in the energy throughput with increase in node mobility can be attributed to the relatively larger amount of energy spent for broadcast tree discoveries. The relatively higher energy throughput while using DMEF can be attributed to the tendency of the broadcast strategy to involve only relatively slow 
moving nodes to be part of the trees. As a result, less energy consumed for broadcast tree discoveries. Under both flooding and DMEF, for a given node mobility and multicast group size, the decrease in energy throughput with increase in network density is more for MAODV and NR-MLPBR, relatively lower for R-MLPBR and is the least for BEMRP. Also, for both flooding and DMEF, for a given network density and node mobility, as the multicast group size is increased from 2 to 4 , the energy throughput decreased by $40-45 \%$ and as the multicast group size is increased from 2 to 24 , the energy throughput decreased by $78-85 \%$.

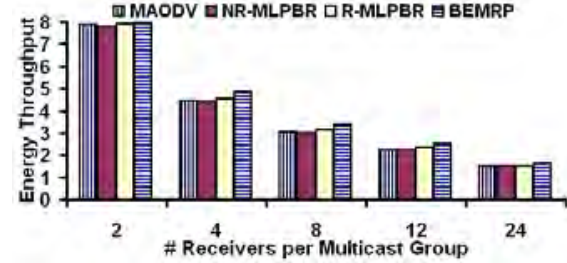

Figure 21.1. 25 nodes, $10 \mathrm{~m} / \mathrm{s}$

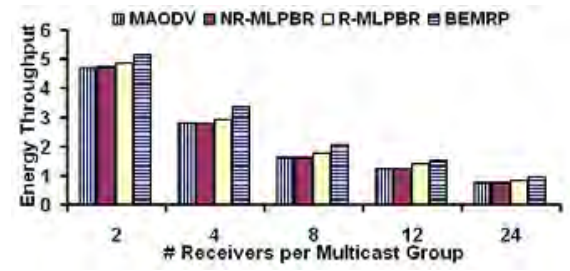

Figure 21.4. 50 nodes, $10 \mathrm{~m} / \mathrm{s}$

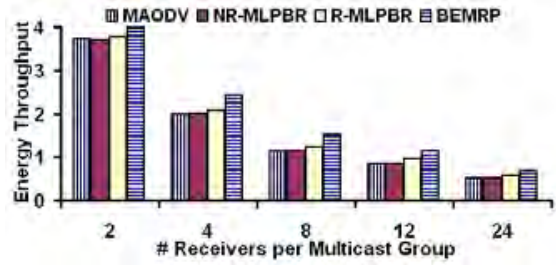

Figure 21.7. 75 nodes, $10 \mathrm{~m} / \mathrm{s}$

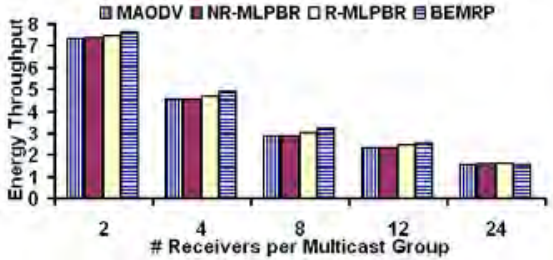

Figure 21.2. 25 nodes, $30 \mathrm{~m} / \mathrm{s}$

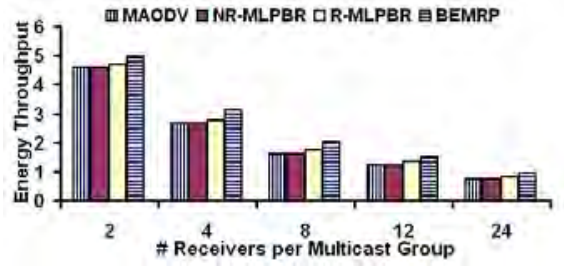

Figure 21.5. 50 nodes, $30 \mathrm{~m} / \mathrm{s}$

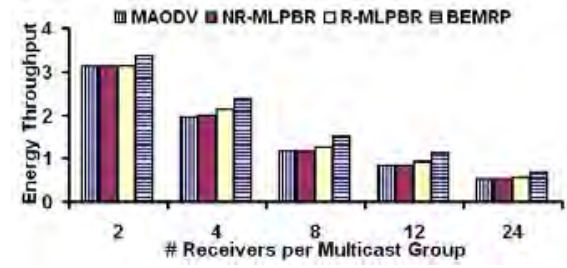

Figure 21.8. 75 nodes, $30 \mathrm{~m} / \mathrm{s}$

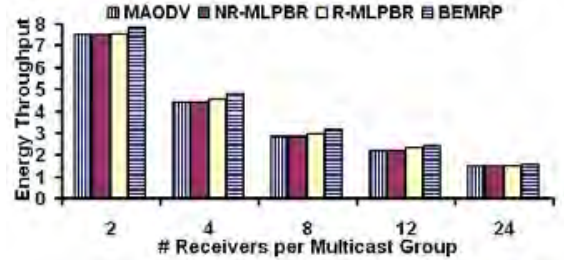

Figure 21.3. 25 nodes, $50 \mathrm{~m} / \mathrm{s}$

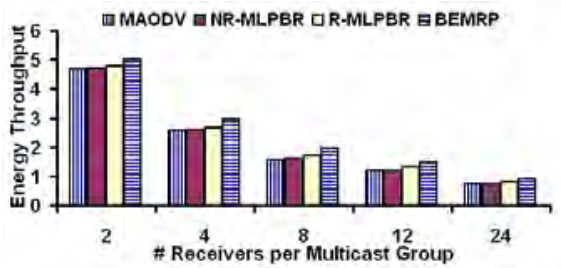

Figure 21.6. 50 nodes, $50 \mathrm{~m} / \mathrm{s}$

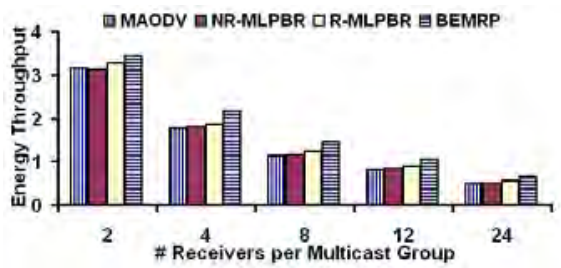

Figure 21.9. 75 nodes, $50 \mathrm{~m} / \mathrm{s}$

Figure 21. Energy Throughput: \# Packets Delivered per Joule (Tree Discovery Procedure: DMEF)

5.6 Average Energy Consumed per Tree Discovery: For a given broadcast strategy, the energy consumed per tree discovery is the same for all the four multicast routing protocols. For both flooding and DMEF, the energy consumed per tree discovery increases with increase in network density, attributed to the involvement of multiple nodes in the broadcast of the MTRMs. For a given node mobility and network density, energy consumed per tree discovery using flooding is $10-37 \%$ more than that of the energy consumed per tree discovery using DMEF. For a given multicast group size, the energy consumed while using flooding in moderate (50 nodes) and high density (75 nodes) networks is respectively about 3.8 and 8 times more than that incurred in networks of low density. This indicates that as the number of nodes is increased by $x$ times ( $x=2$ for moderate density and $x=3$ for high density), the energy consumed due to flooding increases by $2^{x}$ times. In the case of DMEF, for a given multicast group size, the energy consumed in moderate density networks is about 3.7, 3.5 and 3.2 times more than that observed in low density networks for low, moderate and high node mobility conditions respectively. For a given multicast group size, the energy consumed during DMEF in high-density networks is about 7.8, 7.2 and 6.6 times more than that observed in low-density networks for low, moderate and high node mobility conditions respectively. Thus, the energy consumed while using DMEF does not increase exponentially as observed for flooding. DMEF performs appreciably well in lowering the energy consumed per tree discovery with increase in node mobility and/or increase in network density.

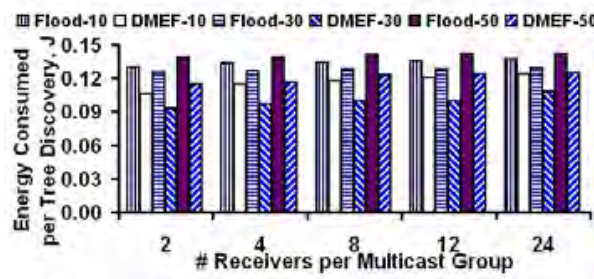

Figure 22.1. 25 nodes

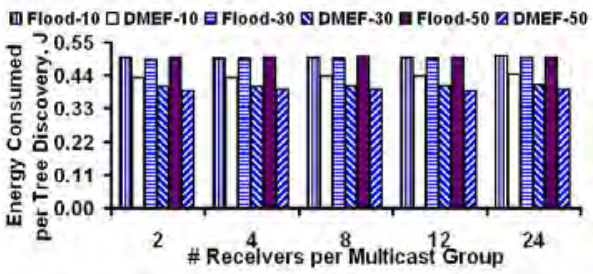

Figure 22.2. 50 nodes

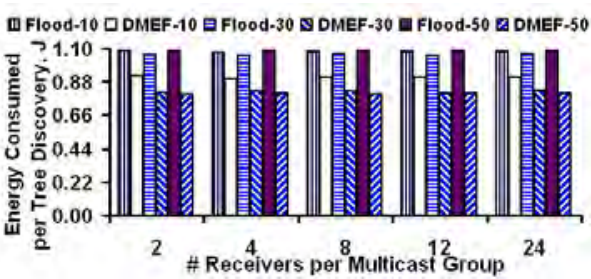

Figure 22.3. 75 nodes

Figure 22. Energy Consumed per Broadcast Tree Discovery: Flooding vs. DMEF 


\section{Conclusions and Future Work}

In this paper, we propose multicast extensions to the location prediction based routing (LPBR) protocol for mobile ad hoc networks (MANETs). LPBR has been proposed to simultaneously minimize the number of route discoveries as well as the hop count of the paths for unicast routing in MANETs. The multicast extensions of LPBR (referred to as NR-MLPBR and R-MLPBR) have been proposed to simultaneously reduce the number of tree discoveries and the hop count per path from the source to each of the receivers of the multicast group. NR-MLPBR and R-MLPBR differ from each other based on the type of path predicted and notified to the source. NR-MLPBR determines the minimum hop path to the source and sends a Multicast Predicted Path Message (MPPM) on the minimum hop path to the source. R-MLPBR assumes that each receiver knows the identity of the other receivers of the multicast group and hence attempts to choose a path that will minimize the number of newly added intermediate nodes to the multicast tree. In pursuit of this, R-MLPBR determines a set of node-disjoint paths to the source on the predicted topology and sends the MPPM on that path that includes the minimum number of non-receiver nodes. If there is a tie, R-MLPBR chooses the path that has the least hop count. The source waits to receive a MPPM from the affected receiver node. R-MLPBR has been thus designed to also reduce the number of links that form the multicast tree, in addition to the source-receiver hop count and the number of global tree discoveries. Nevertheless, R-MLPBR cannot completely nullify the tradeoff between the hop count per source-receiver path and the number of links in the tree. In other words, the number of links per tree discovered using R-MLPBR is still more than that discovered using BEMRP (that aims for minimum number of links in the multicast tree), but the hop count per source-receiver path is significantly smaller than those observed in trees discovered using BEMRP and is the same as that discovered using MAODV (aims to minimize the hop count between source-receiver paths). Simulations have been conducted with both flooding and DMEF as the broadcast tree discovery strategies. Table 2 summarizes the simulation results by listing the protocols that are best suited for optimizing particular performance metric under each of the two broadcast strategies under conditions of low and high network density and node mobility. DMEF helps the multicast routing protocols to discover stable trees and at the same time does not increase the source-receiver hop count appreciably. Hence, the energy consumed per node with DMEF is lower than that incurred with flooding. With the use of DMEF as the tree discovery strategy, the performance of NRMLPBR and R-MLPBR with respect to the time between successive tree discoveries and energy consumed per node actually improved relatively more than that observed for BEMRP and MAODV. This can be attributed to the effective path prediction of the two multicast extensions, an idea inherited from LPBR.

Table 2. Best Multicast Routing Protocols for Different Scenarios and the Two Broadcast Strategies

\begin{tabular}{|c|c|c|c|c|c|}
\hline Performance Metric & $\begin{array}{l}\text { Broadcast } \\
\text { Strategy }\end{array}$ & $\begin{array}{l}\text { Low Density, } \\
\text { Low Mobility }\end{array}$ & $\begin{array}{l}\text { Low Density, } \\
\text { High Mobility }\end{array}$ & $\begin{array}{l}\text { High Density, } \\
\text { Low Mobility }\end{array}$ & $\begin{array}{l}\text { High Density, } \\
\text { High Mobility }\end{array}$ \\
\hline \multirow{2}{*}{$\begin{array}{l}\text { Number of Links per } \\
\text { Multicast Tree }\end{array}$} & Flooding & BEMRP & BEMRP & BEMRP & BEMRP \\
\hline & DMEF & BEMRP & BEMRP & BEMRP & BEMRP \\
\hline \multirow{2}{*}{$\begin{array}{l}\text { Hop Count per Source- } \\
\text { Receiver Path }\end{array}$} & Flooding & $\begin{array}{l}\text { MAODV, NR- } \\
\text { MLPBR, R- } \\
\text { MLPBR }\end{array}$ & $\begin{array}{l}\text { MAODV, NR- } \\
\text { MLPBR, R- } \\
\text { MLPBR }\end{array}$ & $\begin{array}{l}\text { MAODV, NR- } \\
\text { MLPBR, R- } \\
\text { MLPBR }\end{array}$ & $\begin{array}{l}\text { MAODV, NR- } \\
\text { MLPBR, R- } \\
\text { MLPBR }\end{array}$ \\
\hline & DMEF & $\begin{array}{l}\text { MAODV, NR- } \\
\text { MLPBR, R- } \\
\text { MLPBR }\end{array}$ & $\begin{array}{l}\text { MAODV, NR- } \\
\text { MLPBR, R- } \\
\text { MLPBR }\end{array}$ & $\begin{array}{l}\text { MAODV, NR- } \\
\text { MLPBR, R- } \\
\text { MLPBR }\end{array}$ & $\begin{array}{l}\text { MAODV, NR- } \\
\text { MLPBR, R- } \\
\text { MLPBR }\end{array}$ \\
\hline \multirow{2}{*}{$\begin{array}{l}\text { Time between Two Tree } \\
\text { Discoveries }\end{array}$} & Flooding & $\begin{array}{l}\text { BEMRP, R- } \\
\text { MLPBR }\end{array}$ & $\begin{array}{l}\text { NR-MLPBR, } \\
\text { R-MLPBR }\end{array}$ & $\begin{array}{l}\text { R-MLPBR, } \\
\text { BEMRP }\end{array}$ & $\begin{array}{l}\text { R-MLPBR, } \\
\text { BEMRP }\end{array}$ \\
\hline & DMEF & $\begin{array}{l}\text { NR-MLPBR, } \\
\text { R-MLPBR }\end{array}$ & $\begin{array}{l}\text { NR-MLPBR, } \\
\text { R-MLPBR }\end{array}$ & $\begin{array}{l}\text { NR-MLPBR, } \\
\text { R-MLPBR }\end{array}$ & $\begin{array}{l}\text { NR-MLPBR, } \\
\text { R-MLPBR }\end{array}$ \\
\hline \multirow{2}{*}{$\begin{array}{l}\text { Energy Consumed per } \\
\text { Node }\end{array}$} & Flooding & BEMRP & BEMRP & BEMRP & BEMRP \\
\hline & DMEF & BEMRP & BEMRP & BEMRP & BEMRP \\
\hline \multirow{2}{*}{ Energy Throughput } & Flooding & BEMRP & BEMRP & BEMRP & BEMRP \\
\hline & DMEF & BEMRP & BEMRP & BEMRP & BEMRP \\
\hline
\end{tabular}




\section{Appendix}

Table 3. Acronyms and their Expansion

\begin{tabular}{|l|l|}
\hline Acronym & Expansion for the Acronym \\
\hline ABAM & Associativity-Based Ad hoc Multicast Routing Protocol \\
\hline BEMRP & Bandwidth-Efficient Multicast Routing Protocol \\
\hline CBR & Constant Bit Rate \\
\hline CDT & Current Dispatch Time \\
\hline DMEF & Density and Mobility Aware Energy-Efficient Flooding \\
\hline ERS & Expanding Ring Search \\
\hline LPBR & Location Prediction Based Routing Protocol \\
\hline LUV & Location Update Vector \\
\hline MAC & Medium Access Controls \\
\hline MACT & Multicast Activation Message \\
\hline MANET & Mobile Ad hoc Network \\
\hline MAODV & Multicast Extension to the Ad hoc On-demand Distance Vector Routing Protocol \\
\hline MPEM & Multicast Path Error Message \\
\hline MP flag & More Packet flag \\
\hline MPPM & Multicast Predicted Path Message \\
\hline MTEM & Multicast Tree Establishment Message \\
\hline MTRM & Multicast Tree Request Message \\
\hline NEPAT & Next Expected Packet Arrival Time \\
\hline NR-MLPBR & Non-Receiver Aware Multicast Extension to Location Prediction Based Routing Protocol \\
\hline R-MLPBR & Receiver Aware Multicast Extension to Location Prediction Based Routing Protocol \\
\hline RREQ & Route Request Message \\
\hline RREP & Route Reply Message \\
\hline TAT & Tree Acquisition Time \\
\hline TLND & Time Left for Next Dispatch \\
\hline TRT & Tree Repair Time \\
\hline
\end{tabular}

\section{Acknowledgement}

Research was sponsored by the Army Research Laboratory and was accomplished under Cooperative Agreement Number W911NF-08-2-0061. The views and conclusions in this document are those of the authors and should not be interpreted as representing the official policies, either expressed or implied, of the Army Research Laboratory or the U.S. Government. The U.S. Government is authorized to reproduce and distribute reprints for Government purposes notwithstanding any copyright notation herein.

\section{References}

Bettstetter, C., Hartenstein, H. and Perez-Costa, X. 2004. Stochastic Properties of the Random-Waypoint Mobility Model. Wireless Networks, Vol. 10, No. 5, pp. 555-567.

Bianchi, G. 2000. Performance Analysis of the IEEE 802.11 Distributed Coordination Function. IEEE Journal of Selected Areas in Communication, Vol. 18, No. 3, pp. 535-547.

Breslau, L., Estrin, D., Fall, K., Floyd, S., Heidemann, J., Helmy, A., Huang, P., McCanne, S., Varadhan, K., Xu, Y. and Yu, H. 2000. Advances in Network Simulation. IEEE Computer, Vol. 33, No. 5, pp. 59-67.

Broch, J., Maltz, D. A., Johnson, D. B., Hu, Y. C. and Jetcheva, J. 1998. A Performance Comparison of Multi-hop Wireless Ad hoc Network Routing Protocols. Proceedings of the $4^{\text {th }}$ Annual ACM/IEEE Conference on Mobile Computing and Networking, pp. $85-97$.

Cormen, T. H., Leiserson, C. E., Rivest, R. L. and Stein, C. 2001. Introduction to Algorithms. $2^{\text {nd }}$ Edition, MIT Press/ McGraw Hill, New York.

Meghanathan, N. 2008. Stability and Link Efficiency of On-Demand Source Tree-Based Routing Protocols for Mobile Ad Hoc Networks. Proceedings of the International Conference on High Performance Computing, Networking and Communication Systems, pp. 159-166. 
Meghanathan, N. 2009a. A Location Prediction-Based Reactive Routing Protocol to Minimize the Number of Route Discoveries and Hop Count per Path in Mobile Ad Hoc Networks. The Computer Journal, Vol. 52, No. 4, pp. 461-482.

Meghanathan, N. 2009b. A Density and Mobility Aware Energy-Efficient Broadcast Strategy to Determine Stable Routes in Mobile Ad hoc Networks. Proceedings of the International Conference on Wireless Networks, pp. 167-173.

Murthy, C. S. R. and Manoj, B. S. 2004. Ad Hoc Wireless Networks: Architectures and Protocols. Prentice Hall, New Jersey.

Ozaki, T., Kim, J-B. and Suda, T. 2001. Bandwidth-Efficient Multicast Routing for Multihop, Ad hoc Wireless Networks. 2001. Proceedings of the $20^{\text {th }}$ Annual Joint Conference of the IEEE Computer and Communications Societies (INFOCOM), Vol. 2, pp. 1182-1192.

Perkins, C. E. and Royer, E. M. 1999. Ad hoc On-demand Distance Vector Routing. Proceedings of the 2nd Annual IEEE International Workshop on Mobile Computing Systems and Applications, pp. 90-100.

Royer, E. M. and Perkins, C. E. Multicast Operation of the Ad hoc On-demand Distance Vector Routing Protocol. 1999. Proceedings of the $5^{\text {th }}$ ACM/IEEE Annual Conference on Mobile Computing and Networking (MOBICOM), pp. 207-218.

Sheu, J-P., Chao, C-M., Hu, W-K. and Sun, C-W. 2007. A Clock Synchronization Algorithm for Multi-Hop Wireless Ad Hoc Networks. Wireless Personal Communications: An International Journal, Vol. 43, No. 2, pp. 185-200.

Toh, C. K., Guichal, G. and Bunchua, S. 2000. ABAM: On-demand Associativity-based Multicast Routing for Ad hoc Mobile Networks. Proceedings of the $52^{\text {nd }}$ IEEE VTS Fall Vehicular Technology Conference, Vol. 3, pp. 987-993.

Zhou, D. 2005. A Compatible and Scalable Clock Synchronization Protocol in IEEE 802.11 Ad hoc Networks. Proceedings of the International Conference on Parallel Processing, pp. 295-302.

\section{Biographical notes}

Dr. Natarajan Meghanathan is currently working as Assistant Professor of Computer Science at Jackson State University, Mississippi, USA, since August 2005. Dr. Meghanathan received his MS and PhD in Computer Science from Auburn University, AL and The University of Texas at Dallas in August 2002 and May 2005 respectively. Dr. Meghanathan's main areas of research interests are wireless ad hoc networks and sensor networks. He has more than 60 peer-reviewed publications in leading international journals and conferences in this area. Dr. Meghanathan has recently received grants from the U. S. Army Research Laboratory (ARL) and National Science Foundation (NSF). Besides ad hoc networks, Dr. Meghanathan is currently conducting active research in the areas of graph theory, network security and bioinformatics. Dr. Meghanathan has organized workshops and conference sessions in the areas of ad hoc networks, parallel and distributed computing. He serves as the editor of a number of international journals and also in the program committee and organization committees of several leading international conferences in the area of networks. He is listed in the Marquis' Who is Who in America for 2009.

Received June 2010

Accepted July 2010

Final acceptance in revised form August 2010 